Construction of normalized B-splines for a family of smooth spline spaces over Powell-Sabin triangulations

\author{
Hendrik Speleers
}

Report TW600, August 2011

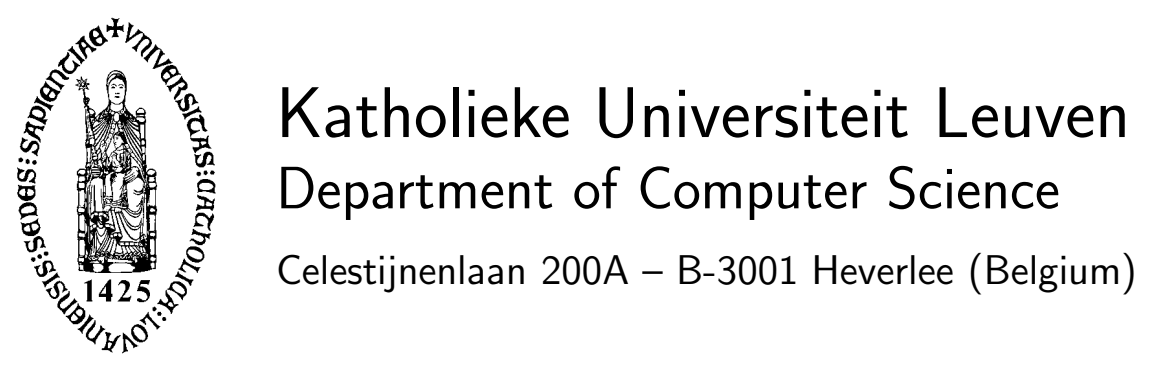




\title{
Construction of normalized B-splines for a family of smooth spline spaces over Powell-Sabin triangulations
}

\author{
Hendrik Speleers \\ Report TW600, August 2011 \\ Department of Computer Science, K.U.Leuven
}

\begin{abstract}
We construct a suitable B-spline representation for a family of bivariate spline functions with smoothness $r \geq 1$ and polynomial degree $3 r-1$. They are defined on a triangulation with PowellSabin refinement. The basis functions have a local support, they are nonnegative and they form a partition of unity. The construction involves the determination of triangles that must contain a specific set of points. We further consider a number of CAGD applications. We show how to define control points and tangent control polynomials (of degree $2 r-1$ ), and we consider the efficient and stable computation of the Bernstein-Bézier form of such splines.
\end{abstract}

Keywords : smooth Powell-Sabin splines, normalized B-splines, macro-elements, control points, control polynomials, Bernstein-Bézier form

MSC : Primary : 65D07 Secondary : 65D17, 41A15 


\title{
Construction of normalized B-splines for a family of smooth spline spaces over Powell-Sabin triangulations
}

\author{
Hendrik Speleers \\ Department of Computer Science, Katholieke Universiteit Leuven \\ Celestijnenlaan 200A, B-3001 Leuven, Belgium
}

\begin{abstract}
We construct a suitable B-spline representation for a family of bivariate spline functions with smoothness $r \geq 1$ and polynomial degree $3 r-1$. They are defined on a triangulation with Powell-Sabin refinement. The basis functions have a local support, they are nonnegative and they form a partition of unity. The construction involves the determination of triangles that must contain a specific set of points. We further consider a number of CAGD applications. We show how to define control points and tangent control polynomials (of degree $2 r-1$ ), and we consider the efficient and stable computation of the Bernstein-Bézier form of such splines. Keywords: smooth Powell-Sabin splines, normalized B-splines, macro-elements, control points, control polynomials, Bernstein-Bézier form
\end{abstract}

\section{Introduction}

A number of authors studied the construction of smooth (finite element) spline spaces defined on arbitrary triangulations. Such a spline space usually has the following properties:

- a certain order of global smoothness, and possibly local supersmoothness;

- a dimension which can be simply expressed in terms of geometrically interesting characteristics of the triangulation (like the number of vertices, edges and/or triangles);

- a linearly independent and stable basis;

- full approximation power.

We refer to [3], and references quoted therein, for an overview of such spline spaces. For the construction of smooth splines with a low polynomial degree one often considers triangulations with a particular macro-structure. Each triangle in the triangulation is then split into a number of subtriangles. The Clough-Tocher split (into three subtriangles) and the Powell-Sabin split (into six subtriangles) are commonly used splits. Splines defined on such refined triangulations are referred to as Clough-Tocher splines and Powell-Sabin splines, respectively.

For Powell-Sabin splines with smoothness $r=1$ and polynomial degree $d=2$, Dierckx [1] has developed an interesting normalized B-spline representation, in which the locally supported basis functions form a convex partition of unity. The spline coefficients in this representation have a geometric meaning, and allow a natural definition of control triangles which are a useful tool for computer aided geometric design. This normalized B-spline representation has been effective in a 
wide range of application areas (see, e.g., $[4,5,13,14])$. Recently, a basis with similar properties has been constructed for Powell-Sabin splines with smoothness $r=2$ and degree $d=5$, see [11], and for (reduced) Clough-Tocher splines with smoothness $r=1$ and degree $d=3$, see [12]. In this paper, we will generalize this construction of a normalized spline basis towards a family of spline spaces with general smoothness $r \geq 1$. These spline spaces are defined on triangulations with Powell-Sabin refinement, and have a polynomial degree $d=3 r-1$.

The paper is organized as follows. In Section 2 we recall some preliminary concepts of bivariate polynomials in Bernstein-Bézier form defined on triangles. In the next section we define a particular family of smooth spline spaces over triangulations of Powell-Sabin's type. Section 4 is devoted to the construction of a normalized basis for these spline spaces. The construction is based on determining a set of triangles that must contain a specific set of points. In Section 5 we define control points and control polynomials which can be useful for modelling purposes. We also show how the Bernstein-Bézier form of such a spline can be computed using only convex combinations. This leads to a stable algorithm for the evaluation of such a spline in its normalized B-spline representation. In Section 6 we consider a family of reduced spline spaces. Finally, we end with some concluding remarks in Section 7.

\section{Bivariate polynomials on triangles}

Let $\mathbb{P}_{d}$ denote the linear space of bivariate polynomials of total degree less than or equal to $d$. In this section we recall the Bernstein-Bézier representation of a polynomial $p_{d} \in \mathbb{P}_{d}$, and some properties of the blossom of such a polynomial.

\subsection{Bernstein-Bézier representation of polynomials}

Let $\mathcal{T}\left(V_{1}, V_{2}, V_{3}\right)$ be a non-degenerate triangle. Any point $P$ in the plane of the triangle can be uniquely expressed in terms of the barycentric coordinates $\tau=\left(\tau_{1}, \tau_{2}, \tau_{3}\right)$ with respect to $\mathcal{T}$, such that

$$
P=\sum_{i=1}^{3} \tau_{i} V_{i}, \quad \text { and } \quad \tau_{1}+\tau_{2}+\tau_{3}=1
$$

Given two points $P^{1}$ and $P^{2}$, the barycentric coordinates $\delta=\left(\delta_{0}, \delta_{1}, \delta_{3}\right)$ of the vector $P^{2}-P^{1}$ with respect to $\mathcal{T}$ are defined as the difference of the barycentric coordinates of both points. If the Euclidean distance $\left\|P^{2}-P^{1}\right\|_{2}=1$, then $\delta$ is called a unit barycentric direction.

Any polynomial $p_{d} \in \mathbb{P}_{d}$ on triangle $\mathcal{T}$ has a unique Bernstein-Bézier representation

$$
p_{d}(P)=p_{d}(\tau)=\sum_{i_{1}+i_{2}+i_{3}=d} b_{i_{1} i_{2} i_{3}} B_{i_{1} i_{2} i_{3}}^{d}(\tau) .
$$

Here,

$$
B_{i_{1} i_{2} i_{3}}^{d}(\tau)=\frac{d !}{i_{1} ! i_{2} ! i_{3} !}\left(\tau_{1}\right)^{i_{1}}\left(\tau_{2}\right)^{i_{2}}\left(\tau_{3}\right)^{i_{3}}
$$

are the Bernstein basis polynomials of degree $d$. The coefficients $b_{i_{1} i_{2} i_{3}}$ are called Bézier ordinates. The Bézier domain points $\xi_{i_{1} i_{2} i_{3}}$ are defined as the points with barycentric coordinates $\left(\frac{i_{1}}{d}, \frac{i_{2}}{d}, \frac{i_{3}}{d}\right)$. By associating the Bézier ordinates $b_{i_{1} i_{2} i_{3}}$ with the Bézier domain points $\xi_{i_{1} i_{2} i_{3}}$, one can display the Bernstein-Bézier representation schematically. The piecewise linear interpolant of the Bézier control points, defined as $\mathbf{b}_{i_{1} i_{2} i_{3}}=\left(\xi_{i_{1} i_{2} i_{3}}, b_{i_{1} i_{2} i_{3}}\right)$, is called the Bézier control net. This control net is tangent to the polynomial surface at the three vertices. 
The disk $D_{r}$ of radius $r$ around vertex $V_{1}$ of $\mathcal{T}$ is the set of domain points defined by

$$
D_{r}\left(V_{1}\right)=\left\{\xi_{i_{1} i_{2} i_{3}}: i_{1} \geq d-r\right\} .
$$

Given a triangulation $\Delta$, then the disk $D_{r}\left(V_{1}\right)$ in $\Delta$ is defined as the set of all domain points in (2.4) for each triangle in $\Delta$ having $V_{1}$ as a vertex. The row $E_{r}$ at distance $r$ parallel to edge $e_{12}=\left\langle V_{1}, V_{2}\right\rangle$ in $\mathcal{T}$ is the set of domain points defined by

$$
E_{r}\left(e_{12}\right)=\left\{\xi_{i_{1} i_{2} i_{3}}: i_{3}=r\right\} .
$$

In the sequel, if we refer to a Bézier ordinate in a disk or on a row, then we actually mean a Bézier ordinate whose corresponding domain point is in that location.

Smoothness conditions are often required between adjoining polynomial patches. Consider a second polynomial $\tilde{p}_{d}$ defined on the domain triangle $\tilde{\mathcal{T}}\left(V_{4}, V_{3}, V_{2}\right)$. Let $\sigma=\left(\sigma_{1}, \sigma_{2}, \sigma_{3}\right)$ be the barycentric coordinates of point $V_{4}$ with respect to triangle $\mathcal{T}\left(V_{1}, V_{2}, V_{3}\right)$. In order to get $C^{\mu}$-continuity between $p_{d}$ and $\tilde{p}_{d}$ across the edge $V_{2}-V_{3}$, the Bézier ordinates $\tilde{b}_{i_{1} i_{2} i_{3}}$ of $\tilde{p}_{d}$ must satisfy the conditions

$$
\tilde{b}_{m, i_{2}, i_{3}}=\sum_{j_{1}+j_{2}+j_{3}=m} b_{j_{1}, i_{3}+j_{2}, i_{2}+j_{3}} B_{j_{1} j_{2} j_{3}}^{m}(\sigma),
$$

for each $m=0, \ldots, \mu$, and $i_{2}+i_{3}=d-m$. We refer to $[2,3]$ for more details.

\subsection{The blossom of polynomials}

The blossom (or polar form) of a polynomial $p_{d}(\tau)$, denoted by $\mathcal{P}_{d}\left(\tau^{1}, \tau^{2}, \ldots, \tau^{d}\right)$, is completely characterized by the following three properties (see, e.g., $[7,10]$ for more details):

Property 2.1. The blossom is symmetric with respect to any permutation $\pi$ of its $d$ arguments,

$$
\mathcal{P}_{d}\left(\tau^{1}, \tau^{2}, \ldots, \tau^{d}\right)=\mathcal{P}_{d}\left(\pi\left(\tau^{1}, \tau^{2}, \ldots, \tau^{d}\right)\right) .
$$

Property 2.2. The blossom is multi-affine, i.e. affine in each of its $d$ arguments. For example,

$$
\mathcal{P}_{d}\left(a \tau+b \sigma, \tau^{2}, \ldots, \tau^{d}\right)=a \mathcal{P}_{d}\left(\tau, \tau^{2}, \ldots, \tau^{d}\right)+b \mathcal{P}_{d}\left(\sigma, \tau^{2}, \ldots, \tau^{d}\right),
$$

for $a+b=1$.

Property 2.3. The restriction of the blossom to the diagonal gives the polynomial $p_{d}$,

$$
p_{d}(\tau)=\mathcal{P}_{d}(\tau, \tau, \ldots, \tau) .
$$

Let $\tau^{l}=\left(\tau_{1}^{l}, \tau_{2}^{l}, \tau_{3}^{l}\right)$ for $l=1, \ldots, d$. One can verify that the blossom of $p_{d}$ can be written as

$$
\mathcal{P}_{d}\left(\tau^{1}, \tau^{2}, \ldots, \tau^{d}\right)=\sum_{i_{1}+i_{2}+i_{3}=d} b_{i_{1} i_{2} i_{3}} \sum_{\pi \in \Pi_{i_{1} i_{2} i_{3}}^{d}} \prod_{l=1}^{d} \tau_{\pi(l)}^{l},
$$

where $b_{i_{1} i_{2} i_{3}}$ are the Bézier ordinates of $p_{d}$ given in $(2.2)$, and $\Pi_{i_{1} i_{2} i_{3}}^{d}$ is the set of all unique permutations of

$$
(\underbrace{1, \ldots, 1}_{i_{1} \text { times }}, \underbrace{2, \ldots, 2}_{i_{2} \text { times }}, \underbrace{3, \ldots, 3}_{i_{3} \text { times }}) .
$$

Note that multi-set $(2.8)$ has $\frac{d !}{i_{1} ! i_{2} ! i_{3} !}$ unique permutations, and each $\pi \in \Pi_{i_{1} i_{2} i_{3}}^{d}$ consists of $d$ elements. 
The multi-affine de Casteljau algorithm can be used for the evaluation of the blossom. The de Casteljau algorithm computes $\mathcal{P}_{d}\left(\tau^{1}, \tau^{2}, \ldots, \tau^{d}\right)$ as follows:

$$
\mathcal{P}_{d}\left(\tau^{1}, \tau^{2}, \ldots, \tau^{d}\right)=b_{0,0,0}^{[d]},
$$

where

for $i_{1}+i_{2}+i_{3}=d$, and

$$
b_{i_{1}, i_{2}, i_{3}}^{[0]}=b_{i_{1} i_{2} i_{3}},
$$

$$
b_{i_{1}, i_{2}, i_{3}}^{[m]}=\tau_{1}^{m} b_{i_{1}+1, i_{2}, i_{3}}^{[m-1]}+\tau_{2}^{m} b_{i_{1}, i_{2}+1, i_{3}}^{[m-1]}+\tau_{3}^{m} b_{i_{1}, i_{2}, i_{3}+1}^{[m-1]},
$$

for $i_{1}+i_{2}+i_{3}=d-m$, and $m=1, \ldots, d$. This algorithm allows us to evaluate the blossom at points inside the domain triangle in a stable way, using only convex combinations.

One can also use the blossom to find the Bernstein-Bézier form of a polynomial $p_{d}$ with respect to another (usually finer) triangle. Let $\mathcal{T}\left(V_{1}, V_{2}, V_{3}\right)$ be a given triangle, and let $b_{i_{1} i_{2} i_{3}}, i_{1}+i_{2}+i_{3}=d$, be the Bézier ordinates of $p_{d}$ with respect to $\mathcal{T}$. Consider a second triangle, whose vertices $W_{l}$, $l=1,2,3$, have $\sigma^{l}=\left(\sigma_{1}^{l}, \sigma_{2}^{l}, \sigma_{3}^{l}\right)$ as barycentric coordinates with respect to the former triangle $\mathcal{T}$. The Bézier ordinates $d_{i_{1} i_{2} i_{3}}$ of $p_{d}$ related to the new triangle can be compactly written as

$$
d_{i_{1} i_{2} i_{3}}=\mathcal{P}_{d}(\underbrace{\sigma^{1}, \ldots, \sigma^{1}}_{i_{1} \text { times }}, \underbrace{\sigma^{2}, \ldots, \sigma^{2}}_{i_{2} \text { times }}, \underbrace{\sigma^{3}, \ldots, \sigma^{3}}_{i_{3} \text { times }}),
$$

for all $i_{1}+i_{2}+i_{3}=d$. If the new triangle is a smaller triangle inside the original domain triangle $\mathcal{T}$, then expression (2.10) is a convex combination of the original ordinates, for any choice $i_{1}+i_{2}+i_{3}=d$. In this way the blossom can be used for subdividing the polynomial onto a triangular partition of $\mathcal{T}$.

Blossoming can also be used to describe directional derivatives of a polynomial (2.2). The $m$-th order (mixed) directional derivative of polynomial $p_{d}$ with respect to the unit barycentric directions $\delta^{i}, i=1, \ldots, m$, related to triangle $\mathcal{T}$ can be compactly expressed as

$$
D_{\delta^{1}, \ldots, \delta^{m}}^{m} p_{d}(\tau)=\frac{d !}{(d-m) !} \mathcal{P}_{d}(\underbrace{\tau, \tau, \ldots, \tau}_{d-m \text { times }}, \delta^{1}, \ldots, \delta^{m}),
$$

where the $m$ barycentric directions $\delta^{i}$ and $(d-m)$ times the barycentric coordinates $\tau$ are taken as the arguments of the blossom.

We end this section by formulating three theorems.

Theorem 2.1. Let $\tau^{l}=\left(\tau_{1}^{l}, \tau_{2}^{l}, \tau_{3}^{l}\right), l=1, \ldots, d$, be barycentric coordinates with respect to a triangle $\mathcal{T}_{1}$, and let $\mathcal{P}_{d}\left(\tau^{1}, \tau^{2}, \ldots, \tau^{d}\right)$ be the blossom of a given polynomial $p_{d}$ defined on $\mathcal{T}_{1}$. The Bézier ordinates of $p_{d}$ are denoted by $b_{i_{1} i_{2} i_{3}}, i_{1}+i_{2}+i_{3}=d$. If these ordinates can be expressed in terms of a blossom $\mathcal{Q}_{d}$ related to another triangle $\mathcal{T}_{2}$,

$$
b_{i_{1} i_{2} i_{3}}=\mathcal{Q}_{d}(\underbrace{\sigma^{1}, \ldots, \sigma^{1}}_{i_{1} \text { times }}, \underbrace{\sigma^{2}, \ldots, \sigma^{2}}_{i_{2} \text { times }}, \underbrace{\sigma^{3}, \ldots, \sigma^{3}}_{i_{3} \text { times }}),
$$

where $\sigma^{l}=\left(\sigma_{1}^{l}, \sigma_{2}^{l}, \sigma_{3}^{l}\right), l=1,2,3$, are given barycentric coordinates with respect to $\mathcal{T}_{2}$, then

$$
\mathcal{P}_{d}\left(\tau^{1}, \tau^{2}, \ldots, \tau^{d}\right)=\mathcal{Q}_{d}\left(\sum_{l=1}^{3} \tau_{l}^{1} \sigma^{l}, \sum_{l=1}^{3} \tau_{l}^{2} \sigma^{l}, \ldots, \sum_{l=1}^{3} \tau_{l}^{d} \sigma^{l}\right) .
$$


Proof. The blossom $\mathcal{P}_{d}\left(\tau^{1}, \tau^{2}, \ldots, \tau^{d}\right)$ can be evaluated using the multi-affine de Casteljau algorithm. By $(2.9 \mathrm{~b})$ and $(2.12)$ we have

$$
b_{i_{1}, i_{2}, i_{3}}^{[0]}=\mathcal{Q}_{d}(\underbrace{\sigma^{1}, \ldots, \sigma^{1}}_{i_{1} \text { times }}, \underbrace{\sigma^{2}, \ldots, \sigma^{2}}_{i_{2} \text { times }}, \underbrace{\sigma^{3}, \ldots, \sigma^{3}}_{i_{3} \text { times }}),
$$

for all $i_{1}+i_{2}+i_{3}=d$. After one step in the de Casteljau algorithm we obtain

$$
b_{i_{1}, i_{2}, i_{3}}^{[1]}=\tau_{1}^{1} b_{i_{1}+1, i_{2}, i_{3}}^{[0]}+\tau_{2}^{1} b_{i_{1}, i_{2}+1, i_{3}}^{[0]}+\tau_{3}^{1} b_{i_{1}, i_{2}, i_{3}+1}^{[0]},
$$

for all $i_{1}+i_{2}+i_{3}=d-1$, and using the multi-affinity and symmetry property of blossom $\mathcal{Q}_{d}$ this can be written as

$$
b_{i_{1}, i_{2}, i_{3}}^{[1]}=\mathcal{Q}_{d}(\tau_{1}^{1} \sigma^{1}+\tau_{2}^{1} \sigma^{2}+\tau_{3}^{1} \sigma^{3}, \underbrace{\sigma^{1}, \ldots, \sigma^{1}}_{i_{1} \text { times }}, \underbrace{\sigma^{2}, \ldots, \sigma^{2}}_{i_{2} \text { times }}, \underbrace{\sigma^{3}, \ldots, \sigma^{3}}_{i_{3} \text { times }}) .
$$

Repeating the same argument for each step in the de Casteljau algorithm, we get

$$
b_{i_{1}, i_{2}, i_{3}}^{[m]}=\mathcal{Q}_{d}(\sum_{l=1}^{3} \tau_{l}^{1} \sigma^{l}, \ldots, \sum_{l=1}^{3} \tau_{l}^{m} \sigma^{l}, \underbrace{\sigma^{1}, \ldots, \sigma^{1}}_{i_{1} \text { times }}, \underbrace{\sigma^{2}, \ldots, \sigma^{2}}_{i_{2} \text { times }}, \underbrace{\sigma^{3}, \ldots, \sigma^{3}}_{i_{3} \text { times }}),
$$

for $i_{1}+i_{2}+i_{3}=d-m$ and $m=1, \ldots, d$. Equality (2.13) follows from the last step in the de Casteljau algorithm, $\mathcal{P}_{d}\left(\tau^{1}, \tau^{2}, \ldots, \tau^{d}\right)=b_{0,0,0}^{[d]}$.

Theorem 2.2. Let $\tau^{l}=\left(\tau_{1}^{l}, \tau_{2}^{l}, \tau_{3}^{l}\right), l=1, \ldots, d$, be barycentric coordinates with respect to a triangle $\mathcal{T}_{1}$, and let $\mathcal{P}_{d}\left(\tau^{1}, \tau^{2}, \ldots, \tau^{d}\right)$ be the blossom of a given polynomial $p_{d}$ defined on $\mathcal{T}_{1}$. The Bézier ordinates of $p_{d}$ are denoted by $b_{i_{1} i_{2} i_{3}}, i_{1}+i_{2}+i_{3}=d$. Suppose that some of these ordinates can be expressed in terms of a blossom $\mathcal{Q}_{d}$ related to another triangle $\mathcal{T}_{2}$ :

$$
b_{i_{1}+m, i_{2}, i_{3}}=\mathcal{Q}_{d}(\underbrace{\sigma^{1}, \ldots, \sigma^{1}}_{i_{1} \text { times }}, \underbrace{\sigma^{2}, \ldots, \sigma^{2}}_{i_{2} \text { times }}, \underbrace{\sigma^{3}, \ldots, \sigma^{3}}_{i_{3} \text { times }}, \rho^{1}, \rho^{2}, \ldots, \rho^{m}),
$$

for a given $m<d$ and all $i_{1}+i_{2}+i_{3}=d-m$. Here, $\sigma^{l}=\left(\sigma_{1}^{l}, \sigma_{2}^{l}, \sigma_{3}^{l}\right), l=1,2,3$, and $\rho^{l}=$ $\left(\rho_{1}^{l}, \rho_{2}^{l}, \rho_{3}^{l}\right), l=1, \ldots, m$, are given barycentric coordinates with respect to $\mathcal{T}_{2}$. Then,

$$
\mathcal{P}_{d}(\tau^{1}, \ldots, \tau^{d-m}, \underbrace{e^{1}, \ldots, e^{1}}_{m \text { times }})=\mathcal{Q}_{d}\left(\sum_{l=1}^{3} \tau_{l}^{1} \sigma^{l}, \ldots, \sum_{l=1}^{3} \tau_{l}^{d-m} \sigma^{l}, \rho^{1}, \ldots, \rho^{m}\right),
$$

with $e^{1}=(1,0,0)$.

Proof. We evaluate blossom $\mathcal{P}_{d}$ in (2.15) with the aid of the multi-affine de Casteljau algorithm. Because of the symmetry property of the blossom, we are allowed to use the barycentric coordinates $e^{1}$ in the first $m$ steps of the algorithm. Then, by (2.9b)-(2.9c) and (2.14) we obtain

$$
\begin{aligned}
b_{i_{1}, i_{2}, i_{3}}^{[m]} & =b_{i_{1}+m, i_{2}, i_{3}}^{[0]} \\
& =\mathcal{Q}_{d}(\underbrace{\sigma^{1}, \ldots, \sigma^{1}}_{i_{1} \text { times }}, \underbrace{\sigma^{2}, \ldots, \sigma^{2}}_{i_{2} \text { times }}, \underbrace{\sigma^{3}, \ldots, \sigma^{3}}_{i_{3} \text { times }}, \rho^{1}, \ldots, \rho^{m}),
\end{aligned}
$$


for all $i_{1}+i_{2}+i_{3}=d-m$. We can proceed as in the proof of Theorem 2.1 for the remaining barycentric coordinates $\tau^{l}, l=1, \ldots, d-m$. The next step in the de Casteljau algorithm gives us for each $i_{1}+i_{2}+i_{3}=d-m-1$,

$$
\begin{aligned}
b_{i_{1}, i_{2}, i_{3}}^{[m+1]} & =\tau_{1}^{1} b_{i_{1}+m+1, i_{2}, i_{3}}^{[0]}+\tau_{2}^{1} b_{i_{1}+m, i_{2}+1, i_{3}}^{[0]}+\tau_{3}^{1} b_{i_{1}+m, i_{2}, i_{3}+1}^{[0]} \\
& =\mathcal{Q}_{d}(\sum_{l=1}^{3} \tau_{l}^{1} \sigma^{l}, \underbrace{\sigma^{1}, \ldots, \sigma^{1}}_{i_{1} \text { times }}, \underbrace{\sigma^{2}, \ldots, \sigma^{2}}_{i_{2} \text { times }}, \underbrace{\sigma^{3}, \ldots, \sigma^{3}}_{i_{3} \text { times }}, \rho^{1}, \ldots, \rho^{m}),
\end{aligned}
$$

using the multi-affinity and symmetry property of the blossom. Repeating this argument for each $\tau^{l}, l=2, \ldots, d-m$, we obtain the equality $(2.15)$.

Theorem 2.3. Let $p_{d_{1}}$ be a polynomial of degree $d_{1}$ defined on the triangle $\mathcal{T}_{1}\left(V_{1}, V_{2}, V_{3}\right)$, and let $p_{d_{2}}$ be a polynomial of degree $d_{2}$ defined on the triangle $\mathcal{T}_{2}\left(V_{1}, W_{2}, W_{3}\right)$, where $d_{2} \leq d_{1}$ and

$$
W_{2}=\frac{d_{1}-d_{2}}{d_{1}} V_{1}+\frac{d_{2}}{d_{1}} V_{2}, \quad W_{3}=\frac{d_{1}-d_{2}}{d_{1}} V_{1}+\frac{d_{2}}{d_{1}} V_{3} .
$$

The Bézier ordinates of $p_{d_{1}}$ are denoted by $b_{i_{1} i_{2} i_{3}}, i_{1}+i_{2}+i_{3}=d_{1}$, and the Bézier ordinates of $p_{d_{2}}$ are denoted by $d_{i_{1} i_{2} i_{3}}, i_{1}+i_{2}+i_{3}=d_{2}$. If

$$
D_{x}^{a} D_{y}^{b} p_{d_{1}}\left(V_{1}\right)=\frac{\left(\begin{array}{c}
d_{1} \\
a+b
\end{array}\right)}{\left(\begin{array}{c}
d_{2} \\
a+b
\end{array}\right)}\left(\frac{d_{2}}{d_{1}}\right)^{a+b} D_{x}^{a} D_{y}^{b} p_{d_{2}}\left(V_{1}\right),
$$

for a given value $0 \leq \mu \leq d_{2}$ and all $0 \leq a+b \leq \mu$, then

$$
b_{d_{1}-\mu+i_{1}, i_{2}, i_{3}}=d_{d_{2}-\mu+i_{1}, i_{2}, i_{3}},
$$

for all $i_{1}+i_{2}+i_{3}=\mu$.

Proof. Let $\delta^{x}$ and $\delta^{y}$ be the unit barycentric directions with respect to triangle $\mathcal{T}_{1}$ in the $x$ - and $y$-direction. Let $\epsilon^{x}$ and $\epsilon^{y}$ be the unit barycentric directions with respect to triangle $\mathcal{T}_{2}$ in the $x$ and $y$-direction. Because of the definition of the triangles, we know that

$$
\epsilon^{x}=\frac{d_{1}}{d_{2}} \delta^{x}, \quad \epsilon^{y}=\frac{d_{1}}{d_{2}} \delta^{y} .
$$

The barycentric coordinates of $V_{1}$ are identical with respect to both triangles, and they are denoted by $e^{1}=(1,0,0)$. Let $\mathcal{P}_{d_{1}}$ and $\mathcal{P}_{d_{2}}$ be the blossoms of polynomials $p_{d_{1}}$ and $p_{d_{2}}$, respectively. By (2.11) we have

$$
D_{x}^{a} D_{y}^{b} p_{d_{1}}\left(V_{1}\right)=\frac{d_{1} !}{\left(d_{1}-a-b\right) !} \mathcal{P}_{d_{1}}(\underbrace{e^{1}, e^{1}, \ldots, e^{1}}_{\left(d_{1}-a-b\right) \text { times }}, \underbrace{\delta^{x}, \ldots, \delta^{x}}_{a \text { times }}, \underbrace{\delta^{y}, \ldots, \delta^{y}}_{b \text { times }}),
$$

and by using (2.18) we also have

$$
D_{x}^{a} D_{y}^{b} p_{d_{2}}\left(V_{1}\right)=\frac{d_{2} !}{\left(d_{2}-a-b\right) !}\left(\frac{d_{1}}{d_{2}}\right)^{a+b} \mathcal{P}_{d_{2}}(\underbrace{e^{1}, e^{1}, \ldots, e^{1}}_{\left(d_{2}-a-b\right) \text { times }}, \underbrace{\delta^{x}, \ldots, \delta^{x}}_{a \text { times }}, \underbrace{\delta^{y}, \ldots, \delta^{y}}_{b \text { times }}) .
$$


Because of (2.16), it follows that for all $0 \leq a+b \leq \mu$

$$
\begin{aligned}
& \mathcal{P}_{d_{1}}(\underbrace{e^{1}, e^{1}, \ldots, e^{1}}_{\left(d_{1}-\mu\right) \text { times }},\underbrace{e^{1}, e^{1}, \ldots, e^{1}}_{(\mu-a-b) \text { times }}, \underbrace{\delta^{x}, \ldots, \delta^{x}}_{a \text { times }}, \underbrace{\delta^{y}, \ldots, \delta^{y}}_{b \text { times }}) \\
&=\mathcal{P}_{d_{2}}(\underbrace{e^{1}, e^{1}, \ldots, e^{1}}_{\left(d_{2}-\mu\right) \text { times }}, \underbrace{e^{1}, e^{1}, \ldots, e^{1}}_{(\mu-a-b) \text { times }}, \underbrace{\delta^{x}, \ldots, \delta^{x}}_{a \text { times }}, \underbrace{\delta^{y}, \ldots, \delta^{y}}_{b \text { times }}),
\end{aligned}
$$

and using (2.7) we get for all $0 \leq a+b \leq \mu$

$$
\sum_{i_{1}+i_{2}+i_{3}=\mu}\left(b_{d_{1}-\mu+i_{1}, i_{2}, i_{3}}-d_{d_{2}-\mu+i_{1}, i_{2}, i_{3}}\right) \sum_{\pi \in \Pi_{i_{1} i_{2} i_{3}}^{\mu}} \prod_{l=1}^{\mu-a-b} e_{\pi(l)}^{1} \prod_{l=\mu-a-b+1}^{\mu-b} \delta_{\pi(l)}^{x} \prod_{l=\mu-b+1}^{\mu} \delta_{\pi(l)}^{y}=0 .
$$

We have $\left(\begin{array}{c}\mu+2 \\ 2\end{array}\right)$ linearly independent constraints involving only the Bézier ordinates $b_{d_{1}-\mu+i_{1}, i_{2}, i_{3}}$ and $d_{d_{2}-\mu+i_{1}, i_{2}, i_{3}}$ with $i_{1}+i_{2}+i_{3}=\mu$. This linear system implies (2.17).

\section{A family of smooth spline spaces over PS-triangulations}

Consider a simply connected subset $\Omega \subset \mathbb{R}^{2}$ with polygonal boundary $\partial \Omega$. Let $\Delta$ be a conforming triangulation $\Delta$ of $\Omega$, where no triangle contains a vertex different from its own three vertices. Let $n_{v}, n_{t}$ and $n_{e}$ be the number of vertices, triangles and edges in $\Delta$, respectively.

A Powell-Sabin (PS-) refinement $\Delta^{*}$ of the triangulation $\Delta$ partitions each triangle $\mathcal{T}_{j} \in \Delta$ into six smaller triangles in the following way $[1,6]$ :

1. Choose an interior point $Z_{j}$ in each triangle $\mathcal{T}_{j}$, so that if two triangles $\mathcal{T}_{i}$ and $\mathcal{T}_{j}$ have a common edge, then the line joining $Z_{i}$ and $Z_{j}$ intersects the common edge at a point between its vertices. For instance, one can take $Z_{j}$ as the incentre of $\mathcal{T}_{j}$.

2. Join each point $Z_{j}$ to the vertices of $\mathcal{T}_{j}$.

3. For each edge of the triangle $\mathcal{T}_{j}$

(a) which is common to a triangle $\mathcal{T}_{i}$ : join $Z_{j}$ to the intersection point of that edge and the line $Z_{i}-Z_{j}$.

(b) which belongs to the boundary $\partial \Omega$ : join $Z_{j}$ to an arbitrary point on that edge.

The obtained subtriangles are denoted by $\mathcal{T}^{*} \in \Delta^{*}$.

Given $0 \leq r<d$, the spline space of degree $d$ on $\Delta^{*}$ with global $C^{r}$-continuity is denoted by

$$
S_{d}^{r}\left(\Delta^{*}\right)=\left\{s \in C^{r}(\Omega):\left.s\right|_{\mathcal{T}^{*}} \in \mathbb{P}_{d}, \mathcal{T}^{*} \in \Delta^{*}\right\} .
$$

We consider a particular subspace of $S_{d}^{r}\left(\Delta^{*}\right)$ with additional smoothness around some vertices and edges. Let $\mathcal{V}=\left\{V_{i}\right\}_{i=1}^{n_{v}}$ be the set of vertices in $\Delta$, let $\mathcal{Z}^{*}=\left\{Z_{i}\right\}_{i=1}^{n_{t}}$ be the set of triangle split points in $\Delta^{*}$, and let $\mathcal{E}^{*}$ be the set of all edges in $\Delta^{*}$ that connect a triangle split point $Z_{i}$ to an edge split point. Given $0 \leq r \leq \rho<d$, the space $S_{d}^{r, \rho}\left(\Delta^{*}\right)$ of supersmooth splines on $\Delta^{*}$ is defined by

$$
S_{d}^{r, \rho}\left(\Delta^{*}\right)=\left\{s \in S_{d}^{r}\left(\Delta^{*}\right): s \in C^{\rho}(W), W \in\left(\mathcal{V} \cup \mathcal{Z}^{*}\right) ; s \in C^{\rho}(e), e \in \mathcal{E}^{*}\right\} .
$$

Here, $C^{\rho}(W)$ means that the polynomials on triangles in $\Delta^{*}$ sharing the vertex $W$ have common derivatives up to order $\rho$ at that vertex. Analogously, $C^{\rho}(e)$ means that the polynomials on triangles in $\Delta^{*}$ sharing the edge $e$ have common derivatives up to order $\rho$ along that edge. 


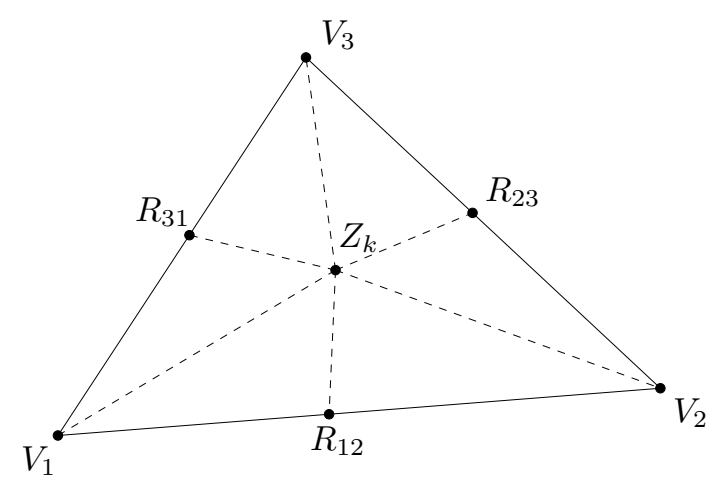

Figure 1: A Powell-Sabin split of a triangle $\mathcal{T}_{k}\left(V_{1}, V_{2}, V_{3}\right)$ drawn with dashed lines.

In this paper we focus on spline spaces $S_{d}^{r, \rho}\left(\Delta^{*}\right)$ of arbitrary smoothness $r \geq 1$, and

$$
d=3 r-1, \quad \rho=2 r-1 .
$$

Such a space will be referred to as Powell-Sabin spline space of smoothness $r$, or PSr-spline space. It will be denoted by $S^{r}\left(\Delta^{*}\right)$. A spline $s(x, y) \in S^{r}\left(\Delta^{*}\right)$ can be defined by means of the following Hermite interpolation problem.

Theorem 3.1. There exists a unique spline $s(x, y) \in S^{r}\left(\Delta^{*}\right)$ satisfying

$$
D_{x}^{a} D_{y}^{b} s\left(V_{l}\right)=f_{x^{a} y^{b}, l}, \quad 0 \leq a+b \leq 2 r-1, \quad l=1, \ldots, n_{v},
$$

and

$$
D_{x}^{a} D_{y}^{b} s\left(Z_{m}\right)=g_{x^{a} y^{b}, m}, \quad 0 \leq a+b \leq r-2, \quad m=1, \ldots, n_{t},
$$

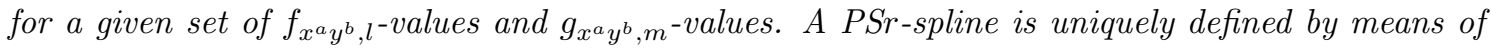
its derivatives up to order $2 r-1$ at the $n_{v}$ vertices $V_{l}$ in $\Delta$ and its derivatives up to order $r-2$ at the $n_{t}$ triangle split points $Z_{m}$ in $\Delta^{*}$.

Proof. We give an explicit construction of the spline $s(x, y) \in S^{r}\left(\Delta^{*}\right)$ satisfying (3.4) in terms of its Bernstein-Bézier representation. We begin by considering a single macro-triangle $\mathcal{T}_{k}\left(V_{1}, V_{2}, V_{3}\right)$ in $\Delta$, as shown in Figure 1. On each of the six subtriangles, the spline is a polynomial of degree $3 r-1$ that can be represented in its Bernstein-Bézier formulation, i.e. with $d=3 r-1$ in equations (2.2) and (2.3). The Bernstein-Bézier representation of $s(x, y)$ on macro-triangle $\mathcal{T}_{k}$ is illustrated in Figure 2 for $r=4$. We now show that the Bézier ordinates in this representation on $\mathcal{T}_{k}$ are uniquely determined by the interpolation conditions (3.4) and the spline smoothness conditions (3.2).

From the Bernstein-Bézier theory and the $C^{2 r-1}$-smoothness at vertex $V_{l}$, we know that the set of derivatives (3.4a) at $V_{l}$ uniquely specifies the Bézier ordinates corresponding to the domain points in the disk $D_{2 r-1}\left(V_{l}\right)$ for each $l=1,2,3$. These three disks are shaded in Figure 2, and their domain points are indicated by the bullets $O$ and

By the $C^{2 r-1}$-smoothness at triangle split point $Z_{k}$ we can regard the Bézier ordinates of $s$ in the disk $D_{2 r-1}\left(Z_{k}\right)$ as the ordinates (after subdivision) of a single polynomial $p$ of degree $2 r-1$ defined on the triangle $\tilde{\mathcal{T}}_{k}\left(W_{1}, W_{2}, W_{3}\right)$ where

$$
W_{i}=\frac{2 r-1}{3 r-1} V_{i}+\frac{r}{3 r-1} Z_{k},
$$




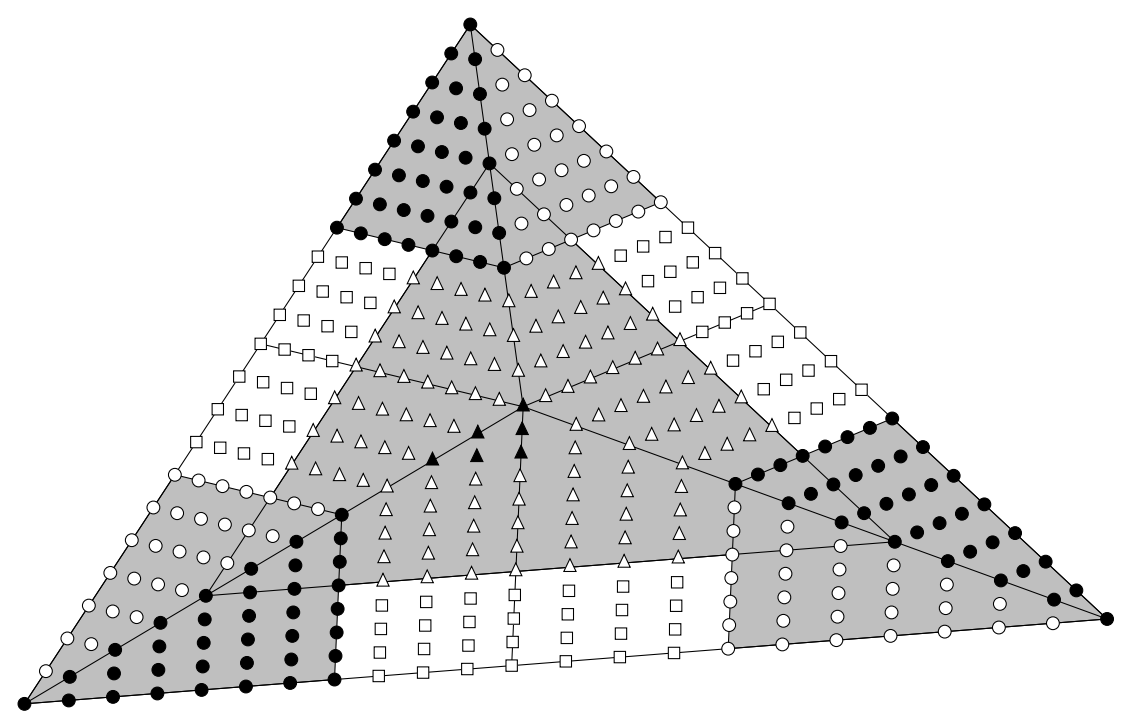

Figure 2: Schematic representation of the Bézier ordinates of a spline $s \in S^{r}$ (with $r=4$ ) on the macro-triangle $\mathcal{T}_{k}$ shown in Figure 1. The points indicated by filled bullets $\bullet$ and filled triangles $\Delta$ form a minimal determining set.

for $i=1,2,3$. The disk $D_{2 r-1}\left(Z_{k}\right)$ is also shaded in Figure 2, and the corresponding domain points are indicated by the triangles $\Delta$ and $\boldsymbol{\Delta}$. Considering only domain points in this disk, we note that the Bézier ordinates related to domain points in $D_{r-1}\left(W_{i}\right), i=1,2,3$, are already determined by our construction. This can be verified in Figure 2 by considering the overlap of the shaded regions. The ordinates related to domain points in $D_{r-2}\left(Z_{k}\right)$ are specified by the interpolation conditions (3.4b). This gives us a total of

$$
3\left(\begin{array}{c}
r+1 \\
2
\end{array}\right)+\left(\begin{array}{l}
r \\
2
\end{array}\right)=\left(\begin{array}{c}
2 r+1 \\
2
\end{array}\right)
$$

independent constraints. They completely specify polynomial $p$, which has degree $2 r-1$ and whose number of degrees of freedom is equal to (3.6).

For each edge $e_{i j}=\left\langle V_{i}, V_{j}\right\rangle$ of $\mathcal{T}_{k}$, the remaining Bézier ordinates on $\mathcal{T}_{k}$ associated with domain points on the rows $0,1, \ldots, r-1$ parallel to $e_{i j}$ are found by using the $C^{2 r-1}$-smoothness conditions across the interior edge $\left\langle R_{i j}, Z_{k}\right\rangle$, with $R_{i j}$ the split point on edge $e_{i j}$, see Figure 1 . These domain points are indicated in Figure 2 by squares $\square$. Fix $0 \leq m \leq r-1$, then the central $4 r-1$ Bézier ordinates on row $E_{m}$ parallel to $e_{12}$ can be regarded as the Bézier ordinates (after subdivision) of a single onedimensional polynomial $\hat{p}$ of degree $2 r-1$ defined over the line segment between the points $\hat{W}_{1}$ and $\hat{W}_{2}$,

$$
\hat{W}_{j}=\frac{2 r-1}{3 r-1} V_{j}+\frac{r-m}{3 r-1} R_{12}+\frac{m}{3 r-1} Z_{k},
$$

$j=1,2$. Of these $4 r-1$ Bézier ordinates, we know that $2 r$ ordinates are already determined by the interpolation conditions (3.4a) at $V_{1}$ and $V_{2}$. This implies that polynomial $\hat{p}$ of degree $2 r-1$ is completely specified as well.

By the above construction, we can uniquely determine the Bézier ordinates on each macro-triangle $\mathcal{T}_{k} \in \Delta$ separately. It remains to check that the $C^{r}$-smoothness conditions across the common edge of any pair of neighbouring macro-triangles in $\Delta$ are satisfied. These conditions, see (2.6), involve 
the Bézier ordinates corresponding to domain points in the rows $0,1, \ldots, r$ parallel to that edge. Let $\mathcal{T}_{1}^{*}\left(V_{1}, R_{12}, Z\right), \mathcal{T}_{2}^{*}\left(V_{2}, R_{12}, Z\right), \mathcal{T}_{3}^{*}\left(V_{1}, R_{12}, \tilde{Z}\right)$ and $\mathcal{T}_{4}^{*}\left(V_{2}, R_{12}, \tilde{Z}\right)$ be the four subtriangles in $\Delta^{*}$ sharing edge $\left\langle V_{1}, V_{2}\right\rangle$. By the construction of the PS-triangulation, we know that $R_{12}$ is the intersection point between the lines $\left\langle V_{1}, V_{2}\right\rangle$ and $\langle Z, \tilde{Z}\rangle$. Let $b_{i_{1} i_{2} i_{3}}^{j}$ be the Bézier ordinates related to subtriangle $\mathcal{T}_{j}^{*}, i_{1}+i_{2}+i_{3}=3 r-1$ and $j=1,2,3,4$. Let $\alpha_{n}^{i_{3}}$ be such that the $C^{r}$-smoothness conditions across $\left\langle V_{1}, R_{12}\right\rangle$ and $\left\langle V_{2}, R_{12}\right\rangle$ are given by

$$
\begin{aligned}
& b_{i_{1} i_{2} i_{3}}^{3}=\sum_{n=0}^{i_{3}} \alpha_{n}^{i_{3}} b_{i_{1}, 3 r-1-n-i_{1}, n}^{1}, \\
& b_{i_{1} i_{2} i_{3}}^{4}=\sum_{n=0}^{i_{3}} \alpha_{n}^{i_{3}} b_{i_{1}, 3 r-1-n-i_{1}, n}^{2},
\end{aligned}
$$

for any $0 \leq i_{3} \leq r$ and $i_{1}+i_{2}+i_{3}=3 r-1$. Note that the weights $\alpha_{n}^{i_{3}}$ are the same in (3.8a) and (3.8b). Because of (3.4a) and the $C^{2 r-1}$-smoothness at the vertices $V_{1}$ and $V_{2}$, the conditions in (3.8) with $i_{1} \geq r$ are automatically satisfied. These conditions connect Bézier ordinates inside disk $D_{2 r-1}\left(V_{1}\right)$ or disk $D_{2 r-1}\left(V_{2}\right)$. We now show that the conditions in (3.8a) are also satisfied for $i_{1}<r$. Because of the $C^{2 r-1}$-smoothness across edge $\left\langle R_{12}, Z\right\rangle$ we can represent $b_{i_{1} i_{2} i_{3}}^{1}$ for any $0 \leq i_{1}<r, 0 \leq i_{3} \leq r$ and $i_{1}+i_{2}+i_{3}=3 r-1$ by

$$
b_{i_{1} i_{2} i_{3}}^{1}=\sum_{m=0}^{r-1} \beta_{m}^{i_{1}} b_{r+m, 2 r-1-m-i_{3}, i_{3}}^{1}+\sum_{m=0}^{r-1} \gamma_{m}^{i_{1}} b_{r+m, 2 r-1-m-i_{3}, i_{3}}^{2}
$$

with particular weights $\beta_{m}^{i_{1}}$ and $\gamma_{m}^{i_{1}}$. Analogously, because of the $C^{2 r-1}$-smoothness across edge $\left\langle R_{12}, \tilde{Z}\right\rangle$ we can represent $b_{i_{1} i_{2} i_{3}}^{3}$ for any $0 \leq i_{1}<r, 0 \leq i_{3} \leq r$ and $i_{1}+i_{2}+i_{3}=3 r-1$ by

$$
b_{i_{1} i_{2} i_{3}}^{3}=\sum_{m=0}^{r-1} \beta_{m}^{i_{1}} b_{r+m, 2 r-1-m-i_{3}, i_{3}}^{3}+\sum_{m=0}^{r-1} \gamma_{m}^{i_{1}} b_{r+m, 2 r-1-m-i_{3}, i_{3}}^{4},
$$

with the same weights $\beta_{m}^{i_{1}}$ and $\gamma_{m}^{i_{1}}$ as in (3.9). We already know that the conditions in (3.8) hold for $i_{1} \geq r$. Thus, combining (3.8)-(3.10), we get for $i_{1}<r$

$$
\begin{aligned}
b_{i_{1} i_{2} i_{3}}^{3} & =\sum_{m=0}^{r-1} \beta_{m}^{i_{1}}\left(\sum_{n=0}^{i_{3}} \alpha_{n}^{i_{3}} b_{r+m, 2 r-1-n-m, n}^{1}\right)+\sum_{m=0}^{r-1} \gamma_{m}^{i_{1}}\left(\sum_{n=0}^{i_{3}} \alpha_{n}^{i_{3}} b_{r+m, 2 r-1-n-m, n}^{2}\right) \\
& =\sum_{n=0}^{i_{3}} \alpha_{n}^{i_{3}}\left(\sum_{m=0}^{r-1} \beta_{m}^{i_{1}} b_{r+m, 2 r-1-n-m, n}^{1}+\sum_{m=0}^{r-1} \gamma_{m}^{i_{1}} b_{r+m, 2 r-1-n-m, n}^{2}\right) \\
& =\sum_{n=0}^{i_{3}} \alpha_{n}^{i_{3}} b_{i_{1}, 3 r-1-n-i_{1}, n}^{1} .
\end{aligned}
$$

Using a similar argument, we can also prove that the conditions in (3.8b) are satisfied for $i_{1}<r$.

We can conclude that the above construction of the Bernstein-Bézier representation of $s(x, y)$ shows that conditions (3.4) are sufficient and necessary to specify (all Bézier ordinates of) a Powell-Sabin spline $s(x, y)$ uniquely on $\Delta^{*}$.

Remark 3.1. By the constructive nature of the proof of Theorem 3.1, one can easily define a stable local minimal determining set of Bézier domain points. A minimal determining set is a minimal subset of Bézier domain points $\xi_{i}$, such that if the corresponding Bézier ordinates $b_{i}$ in the Bernstein-Bézier representation of a spline $s \in S^{r}$ are equal to zero then the spline $s$ must be zero (see [3]). Such a minimal determining set is illustrated in Figure 2. The points in this set are indicated by filled bullets $\bullet$ and filled triangles $\Delta$. 
The dimension of the space $S^{r}\left(\Delta^{*}\right)$ follows immediately from Theorem 3.1.

Theorem 3.2. The dimension of $S^{r}\left(\Delta^{*}\right)$ is given by

$$
\operatorname{dim} S^{r}\left(\Delta^{*}\right)=r(2 r+1) n_{v}+\frac{r(r-1)}{2} n_{t} .
$$

Proof. By Theorem 3.1 we know that the spline space $S^{r}\left(\Delta^{*}\right)$ has $\left(\begin{array}{c}2 r+1 \\ 2\end{array}\right)$ degrees of freedom for each vertex $V_{l}$ and $\left(\begin{array}{c}r \\ 2\end{array}\right)$ degrees of freedom for each triangle split point $Z_{m}$.

The spline space $S^{r}\left(\Delta^{*}\right)$ has also an optimal approximation order. Let $\|\cdot\|_{L_{p}}$ be the $L_{p}$-norm on $\Omega$, and let $|\cdot|_{W_{p}^{k}}$ be the usual semi-norm in the Sobolev space $W_{p}^{k}(\Omega)$, i.e.,

$$
|v|_{W_{p}^{k}}=\left(\sum_{a+b=k}\left\|D_{x}^{a} D_{y}^{b} v\right\|_{L_{p}}^{p}\right)^{1 / p} .
$$

Theorem 3.3. For every $0 \leq k \leq d:=3 r-1$, there exists an interpolation operator $\mathcal{I}^{r}$ : $W_{p}^{k+1}(\Omega) \rightarrow S^{r}\left(\Delta^{*}\right)$ such that

$$
\left\|D_{x}^{a} D_{y}^{b}\left(f-\mathcal{I}^{r} f\right)\right\|_{L_{p}} \leq K|\Delta|^{k+1-a-b}|f|_{W_{p}^{k+1}},
$$

for every function $f \in W_{p}^{k+1}(\Omega)$, with $0 \leq a+b \leq k$ and $|\Delta|$ the mesh size of $\Delta$. If $\Omega$ is convex then the constant $K$ depends only on $r, p, k$ and on the smallest angle in $\Delta^{*}$. If $\Omega$ is non-convex, it also depends on the Lipschitz constant $L_{\partial \Omega}$ associated with the boundary of $\Omega$.

Proof. Using the techniques provided in Chapters 5 and 8 of [3], the result follows immediately from the construction of a stable local minimal determining set (see Remark 3.1).

The spline space $S^{r}\left(\Delta^{*}\right)$ with $r=1(d=2, \rho=1)$ is the well-known quadratic Powell-Sabin spline space, see $[1,6,9]$, and the space with $r=2(d=5, \rho=3)$ is the quintic Powell-Sabin spline space constructed in [11].

\section{Normalized Powell-Sabin B-splines}

In this section we look for a suitable B-spline representation of $s(x, y) \in S^{r}\left(\Delta^{*}\right)$,

$$
s(x, y)=\sum_{i=1}^{n_{v}} \sum_{j_{1}+j_{2}+j_{3}=2 r-1} c_{i, j_{1} j_{2} j_{3}}^{v, r} B_{i, j_{1} j_{2} j_{3}}^{v, r}(x, y)+\sum_{k=1}^{n_{t}} \sum_{j_{1}+j_{2}+j_{3}=r-2} c_{k, j_{1} j_{2} j_{3}}^{t, r} B_{k, j_{1} j_{2} j_{3}}^{t, r}(x, y),
$$

in which the basis functions $B_{i, j_{1} j_{2} j_{3}}^{v, r}(x, y)$ and $B_{k, j_{1} j_{2} j_{3}}^{t, r}(x, y)$ have a local support and form a convex partition of unity, i.e., they are nonnegative and sum up to one. We will call $B_{i, j_{1} j_{2} j_{3}}^{v, r}(x, y)$ and $B_{k, j_{1} j_{2} j_{3}}^{t, r}(x, y)$ a Powell-Sabin B-spline with respect to vertex $V_{i}$ and triangle $\mathcal{T}_{k}$, respectively. We first describe the construction of the Powell-Sabin B-splines, and then we show some properties. 


\subsection{Construction of the basis}

Let $M_{i}$ be the molecule (also called 1-ring or cell) of vertex $V_{i}$, defined as the union of all triangles in $\Delta$ that contain $V_{i}$. Let $N^{v}$ and $N^{t}$ be defined by

$$
N^{v}=\left(\begin{array}{c}
2 r+1 \\
2
\end{array}\right), \quad N^{t}=\left(\begin{array}{l}
r \\
2
\end{array}\right) .
$$

With each vertex $V_{i}$ we associate $N^{v}$ linearly independent sets $A_{i, j_{1} j_{2} j_{3}}$ consisting of $N^{v}$ elements, i.e.,

$$
A_{i, j_{1} j_{2} j_{3}}=\left\{\alpha_{i, j_{1} j_{2} j_{3}}^{a b}, 0 \leq a+b \leq 2 r-1\right\},
$$

for all $j_{1}+j_{2}+j_{3}=2 r-1$. With each triangle $\mathcal{T}_{k}$ we associate $N^{t}$ linearly independent sets $B_{k, j_{1} j_{2} j_{3}}$ consisting of $N^{t}$ elements, i.e.,

$$
B_{k, j_{1} j_{2} j_{3}}=\left\{\beta_{k, j_{1} j_{2} j_{3}}^{a b}, 0 \leq a+b \leq r-2\right\},
$$

for all $j_{1}+j_{2}+j_{3}=r-2$. These sets are constructed as follows.

1. For each vertex $V_{i}$ in $\Delta$, identify the so-called $\mathrm{PS} r$-points. They are defined as

$$
S_{i l}=\frac{r}{3 r-1} V_{i}+\frac{2 r-1}{3 r-1} V_{l}
$$

for all vertices $V_{l}$ that are situated at the boundary of the molecule $M_{i}$ of $V_{i}$. The vertex $V_{i}$ itself is also a $\mathrm{PS} r$-point.

2. For each vertex $V_{i}$, find a triangle $t_{i}\left(Q_{i, 1}, Q_{i, 2}, Q_{i, 3}\right)$ that contains all the PSr-points of $V_{i}$. Denote its vertices by $Q_{i, j}=\left(X_{i, j}, Y_{i, j}\right)$. The triangles $t_{i}, i=1, \ldots, n_{v}$, will be called PSr-triangles.

3. For each vertex $V_{i}$, consider the $N^{v}$ Bernstein basis polynomials (2.3) of degree $2 r-1$ defined

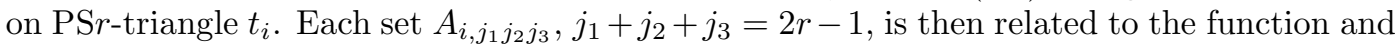
derivative values up to order $2 r-1$ of one of these Bernstein basis polynomials evaluated at the point $V_{i}$ :

$$
\alpha_{i, j_{1} j_{2} j_{3}}^{a b}=\frac{\left(\begin{array}{c}
3 r-1 \\
a+b
\end{array}\right)}{\left(\begin{array}{c}
2 r-1 \\
a+b
\end{array}\right)}\left(\frac{2 r-1}{3 r-1}\right)^{a+b} D_{x}^{a} D_{y}^{b} B_{j_{1} j_{2} j_{3}}^{2 r-1}\left(V_{i}\right)
$$

for all $0 \leq a+b \leq 2 r-1$

4. For each triangle $\mathcal{T}_{k}\left(V_{1}, V_{2}, V_{3}\right)$, consider the $N^{v}$ Bernstein basis polynomials (2.3) of degree $2 r-1$ defined on the triangle with vertices $W_{i}, i=1,2,3$, as given in (3.5). Each set $B_{k, j_{1} j_{2} j_{3}}$, $j_{1}+j_{2}+j_{3}=r-2$, is then related to the function and derivative values up to order $r-2$ of one of these Bernstein basis polynomials evaluated at the split point $Z_{k}$ of macro-triangle $\mathcal{T}_{k}$ :

$$
\beta_{k, j_{1} j_{2} j_{3}}^{a b}=\frac{\left(\begin{array}{c}
3 r-1 \\
a+b
\end{array}\right)}{\left(\begin{array}{c}
2 r-1 \\
a+b
\end{array}\right)}\left(\frac{2 r-1}{3 r-1}\right)^{a+b} D_{x}^{a} D_{y}^{b} B_{r-1-j_{1}, r-1-j_{2}, r-1-j_{3}}^{2 r-1}\left(Z_{k}\right),
$$

for all $0 \leq a+b \leq r-2$. 


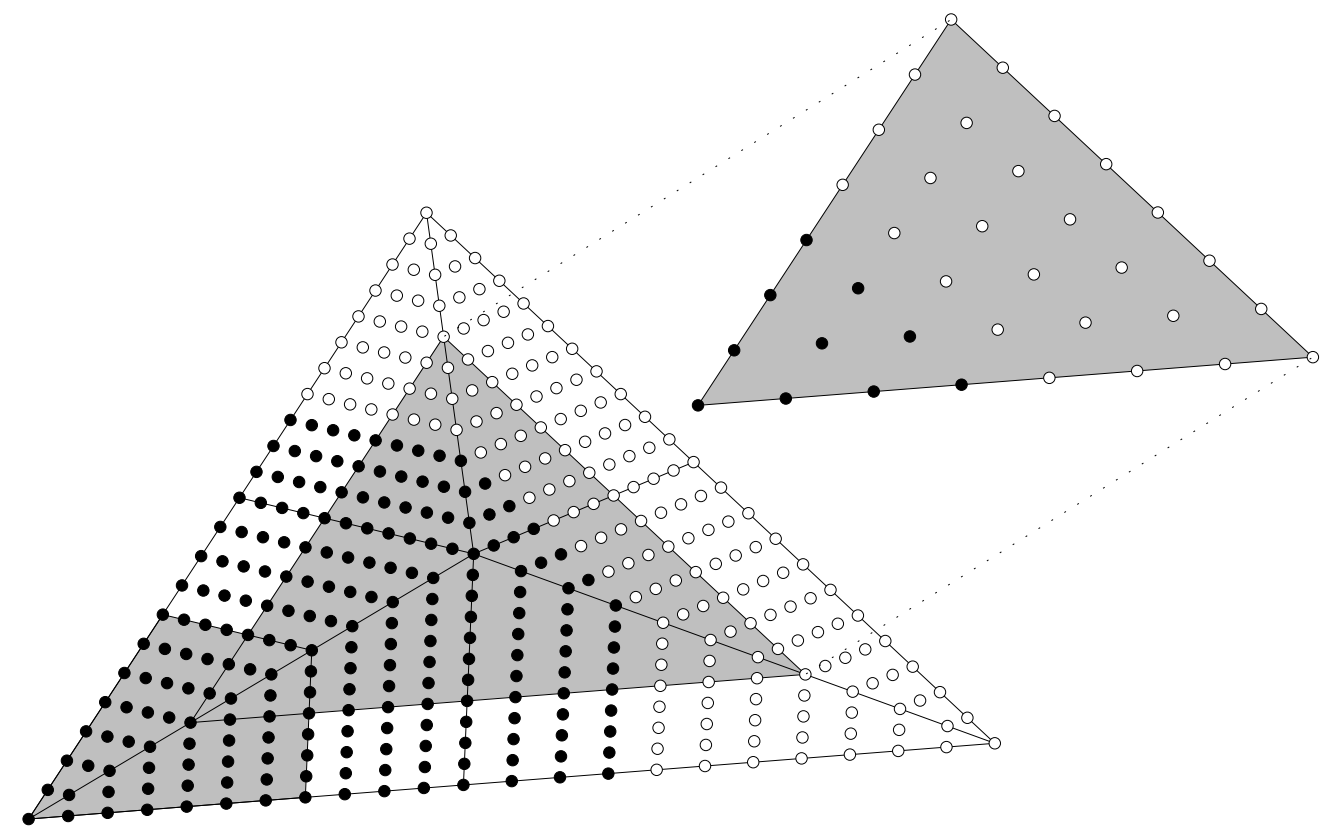

(a)

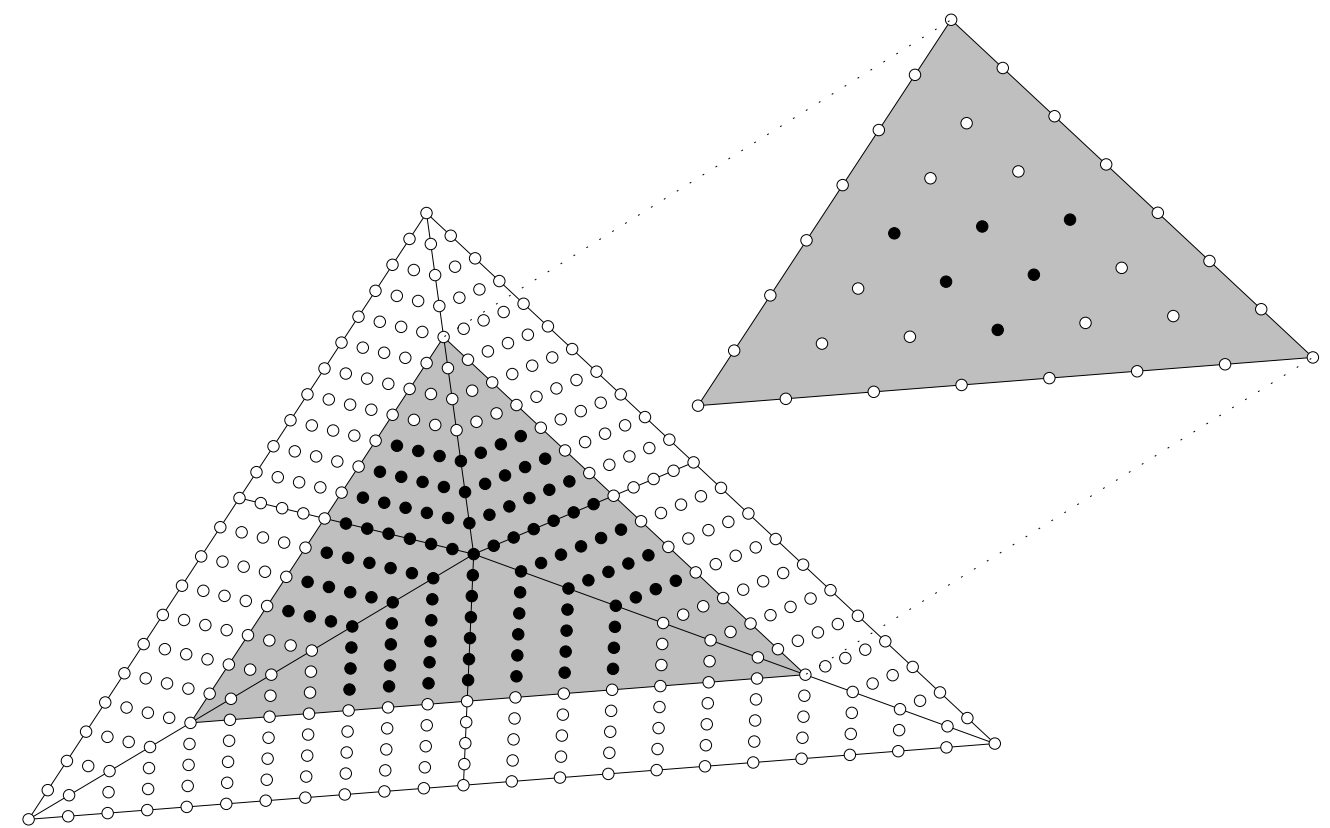

(b)

Figure 3: Schematic representation of the Bézier ordinates of (a) a PSr B-spline with respect to a vertex and (b) a PSr B-spline with respect to a triangle, for $r=4$, on the macro-triangle $\mathcal{T}_{k}$ shown in Figure 1. Bézier ordinates that are known to be zero are indicated by open bullets o; other ordinates are indicated by filled bullets $\bullet$. The Bernstein-Bézier representation of the polynomial $p$ used for the computation of the ordinates corresponding to disk $D_{2 r-1}\left(Z_{k}\right)$ is also given. 
We remark that the PSr-triangles are not uniquely defined. An optimization strategy can be used to select triangles of minimal area, as has been suggested in the case $r=1$ and $r=2$, see $[1,11]$. Constructing such triangles of minimal area is equivalent to solving a quadratic programming problem.

The basis functions $B_{i, j_{1} j_{2} j_{3}}^{v, r}(x, y)$ and $B_{k, j_{1} j_{2} j_{3}}^{t, r}(x, y)$ are then constructed in the following way:

1. The B-spline $B_{i, j_{1} j_{2} j_{3}}^{v, r}(x, y)$ with respect to vertex $V_{i}$ is defined as the unique solution of interpolation problem (3.4) with all $f_{x^{a} y^{b}, l}=0$, except for $l=i$, where $f_{x^{a} y^{b}, i}=\alpha_{i, j_{1} j_{2} j_{3}}^{a b}$, and with all $g_{x^{a} y^{b}, m}=0$, except for any $m$ such that $\mathcal{T}_{m} \in M_{i}$, where $g_{x^{a} y^{b}, m}=\gamma_{i, m, j_{1} j_{2} j_{3}}^{a b}$. The values $\alpha_{i, j_{1} j_{2} j_{3}}^{a b}$ are determined by the PSr-triangle $t_{i}$, see (4.6). The values $\gamma_{i, m, j_{1} j_{2} j_{3}}^{a b}$ are specified with the aid of the Bernstein-Bézier representation of the B-spline as follows.

We consider a macro-triangle $\mathcal{T}_{m}\left(V_{1}, V_{2}, V_{3}\right)$ in the molecule $M_{1}$ of $V_{1}$, as illustrated in Figure 1 , and the B-spline $B_{1, j_{1} j_{2} j_{3}}^{v, r}(x, y)$ associated with vertex $V_{1}$. The corresponding Bézier ordinates are schematically represented in Figure 3(a) for $r=4$. Bézier ordinates, that are known to be zero, are indicated by open bullets $\circ$ in the figure. The other ordinates are indicated by filled bullets $\bullet$. Because of the $C^{2 r-1}$-smoothness at vertex $V_{1}$, the Bézier ordinates in the disk $D_{2 r-1}\left(V_{1}\right)$ are completely determined by the set of values $A_{1, j_{1} j_{2} j_{3}}$. The Bézier ordinates in the disk $D_{2 r-1}\left(Z_{m}\right)$ can be regarded as ordinates (after subdivision) of a single polynomial $p$ of degree $2 r-1$ defined on the triangle with vertices $W_{i}, i=1,2,3$, given in (3.5). The polynomial $p$ is defined on this triangle by means of its Bernstein-Bézier representation. Let $b_{i_{1} i_{2} i_{3}}, i_{1}+i_{2}+i_{3}=2 r-1$, be the Bézier ordinates in this representation. All ordinates $b_{i_{1} i_{2} i_{3}}$ with $i_{1}<r$ are taken to be zero. The values of the ordinates $b_{i_{1} i_{2} i_{3}}$ with $i_{1} \geq r$ are uniquely determined by the (subdivision) relation between them and the Bézier ordinates in the disk $D_{2 r-1}\left(V_{1}\right)$ of the B-spline (which are specified by the set $A_{1, j_{1} j_{2} j_{3}}$ ). The zero and nonzero ordinates $b_{i_{1} i_{2} i_{3}}$ are illustrated on the triangle shown on the right in Figure 3(a).

The value of $\gamma_{1, m, j_{1} j_{2} j_{3}}^{a b}$ related to triangle $\mathcal{T}_{m}$ is then obtained by evaluating the polynomial $p$ of degree $2 r-1$ and its derivatives at split point $Z_{m}$ :

$$
\gamma_{1, m, j_{1} j_{2} j_{3}}^{a b}=\frac{\left(\begin{array}{c}
3 r-1 \\
a+b
\end{array}\right)}{\left(\begin{array}{c}
2 r-1 \\
a+b
\end{array}\right)}\left(\frac{2 r-1}{3 r-1}\right)^{a+b} D_{x}^{a} D_{y}^{b} p\left(Z_{m}\right) .
$$

2. The B-spline $B_{k, j_{1} j_{2} j_{3}}^{t, r}(x, y)$ with respect to triangle $\mathcal{T}_{k}$ is defined as the unique solution of interpolation problem (3.4) with all $f_{x^{a} y^{b}, l}=0$ and with all $g_{x^{a} y^{b}, m}=0$, except for $m=k$, where $g_{x^{a} y^{b}, k}=\beta_{k, j_{1} j_{2} j_{3}}^{a b}$. The values $\beta_{k, j_{1} j_{2} j_{3}}^{a b}$ are defined by (4.7).

To illustrate the Bernstein-Bézier representation of this B-spline, we consider again the triangle $\mathcal{T}_{k}\left(V_{1}, V_{2}, V_{3}\right)$ shown in Figure 1 . The corresponding Bézier ordinates are schematically represented in Figure 3(b) for $r=4$. Most of these ordinates are zero. The Bézier ordinates in the disk $D_{2 r-1}\left(Z_{k}\right)$ are found by subdivision of a polynomial $p$ of degree $2 r-1$ defined on the triangle with vertices $W_{i}, i=1,2,3$, given in (3.5). In the Bernstein-Bézier representation of $p$ on this triangle, the Bézier ordinates in the neighbourhood of $W_{i}$ are zero. The corresponding domain points are highlighted on the triangle shown on the right in Figure 3(b).

In Section 5.2 we will provide a more elegant and practical way to compute the Bernstein-Bézier representation of a Powell-Sabin spline $s \in S^{r}\left(\Delta^{*}\right)$ in representation (4.1), and thus also of a normalized B-spline. 


\subsection{Some properties of the Powell-Sabin B-splines}

In this section we mainly focus on the Powell-Sabin B-spline $B_{1, j_{1} j_{2} j_{3}}^{v, r}(x, y)$ with respect to vertex $V_{1}$, and the B-spline $B_{k, j_{1} j_{2} j_{3}}^{t, r}(x, y)$ with respect to triangle $\mathcal{T}_{k}\left(V_{1}, V_{2}, V_{3}\right)$, as shown in Figure 1 . We assume that the points indicated in this figure have the following barycentric coordinates with respect to $\mathcal{T}_{k}$ :

$$
\begin{aligned}
& V_{1}=(1,0,0), \quad V_{2}=(0,1,0), \quad V_{3}=(0,0,1), \quad Z_{k}=\left(z_{1}, z_{2}, z_{3}\right), \\
& R_{12}=\left(\lambda_{12}, \lambda_{21}, 0\right), \quad R_{23}=\left(0, \lambda_{23}, \lambda_{32}\right), \quad R_{31}=\left(\lambda_{13}, 0, \lambda_{31}\right) .
\end{aligned}
$$

We first formulate some lemmata with regard to the Bernstein-Bézier representation of the Bsplines.

Lemma 4.1. The Bézier ordinates corresponding to domain points in the disk $D_{2 r-1}\left(V_{1}\right)$ of a Powell-Sabin B-spline $B_{1, j_{1} j_{2} j_{3}}^{v, r}(x, y)$ in Bernstein-Bézier form can be regarded as the Bézier ordinates (after subdivision) of the Bernstein basis polynomial $B_{j_{1} j_{2} j_{3}}^{2 r-1}(x, y)$ of degree $2 r-1$ defined on the PSr-triangle $t_{1}$ of vertex $V_{1}$. These Bézier ordinates are nonnegative.

Proof. From definition (4.6) and Theorem 2.3 (with $d_{1}=3 r-1$ and $\mu=d_{2}=2 r-1$ ), it follows that the Bézier ordinates in the disk $D_{2 r-1}\left(V_{1}\right)$ of the Powell-Sabin B-spline $B_{1, j_{1} j_{2} j_{3}}^{v, r}(x, y)$ can be regarded as the Bézier ordinates (after subdivision) of a Bernstein basis polynomial defined on the $\mathrm{PS} r$-triangle.

We now prove that these ordinates are nonnegative. To this end, we first show that the domain points in the disk $D_{2 r-1}\left(V_{1}\right)$ are contained in the PSr-triangle $t_{1}$ of vertex $V_{1}$. Let $\mathcal{T}_{k}\left(V_{1}, V_{2}, V_{3}\right)$ be a macro-triangle in the molecule $M_{1}$ of $V_{1}$, as indicated in Figure 1. The domain points inside disk $D_{2 r-1}\left(V_{1}\right)$ in $\mathcal{T}_{k}$ are defined by

$$
\tau_{1} V_{1}+\tau_{2} R_{12}+\tau_{3} Z_{k}, \quad \text { or } \quad \tau_{1} V_{1}+\tau_{2} R_{13}+\tau_{3} Z_{k},
$$

for some $\frac{r}{3 r-1} \leq \tau_{1}, 0 \leq \tau_{2}, 0 \leq \tau_{3}, \tau_{1}+\tau_{2}+\tau_{3}=1$. By the definition of the PSr-triangle $t_{1}$ we know that it contains the triangle spanned by the $\mathrm{PS} r$-points $V_{1}, S_{12}$ and $S_{13}$, see (4.5). It is easy to see that the considered domain points are all inside this triangle. It follows that the barycentric coordinates of these points with respect to the PSr-triangle are nonnegative. We can use a similar argument for all other triangles in the molecule $M_{1}$ of $V_{1}$.

Any Bernstein basis polynomial is nonnegative on its domain triangle. Its blossom $\mathcal{B}_{2 r-1}\left(\tau^{1}, \ldots, \tau^{2 r-1}\right)$ is nonnegative when the barycentric coordinates $\tau^{l}, l=1, \ldots, 2 r-1$, are nonnegative. Applying subdivision rules (2.10), we then obtain that the Bézier ordinates of the Powell-Sabin B-spline $B_{1, j_{1} j_{2} j_{3}}^{v, r}(x, y)$ in the disk $D_{2 r-1}\left(V_{1}\right)$ are nonnegative as well.

Lemma 4.2. Let $\mathcal{T}_{k}\left(V_{1}, V_{2}, V_{3}\right)$ be a triangle in the molecule of vertex $V_{1}$. The Bézier ordinates corresponding to domain points in the disk $D_{2 r-1}\left(Z_{k}\right)$ of a Powell-Sabin B-spline $B_{1, j_{1} j_{2} j_{3}}^{v, r}(x, y)$ related to vertex $V_{1}$ in Bernstein-Bézier form can be regarded as the Bézier ordinates (after subdivision) of a nonnegative polynomial $p$ of degree $2 r-1$ defined over the triangle with vertices $W_{i}$, $i=1,2,3$, as given in (3.5). These Bézier ordinates are nonnegative.

Proof. From the construction of B-spline $B_{1, j_{1} j_{2} j_{3}}^{v, r}(x, y)$ we know that its Bézier ordinates corresponding to domain points in the disk $D_{2 r-1}\left(Z_{k}\right)$ can be regarded as the Bézier ordinates (after

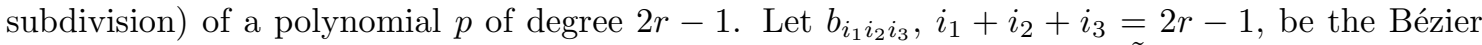
ordinates of this polynomial in Bernstein-Bézier representation on triangle $\tilde{\mathcal{T}}_{k}\left(W_{1}, W_{2}, W_{3}\right)$. In this representation only the $\left(\begin{array}{c}r+1 \\ 2\end{array}\right)$ Bézier ordinates in the neighbourhood of $W_{1}$ are nonzero (see the 
triangle on the right in Figure 3(a)). The values of these nonzero ordinates are uniquely determined by the set $A_{1, j_{1} j_{2} j_{3}}$. We also consider the Bernstein-Bézier form of this polynomial on the subtriangle $\hat{\mathcal{T}}_{k}\left(W_{1}, T_{12}, Z_{k}\right)$, where

$$
T_{12}=\frac{2 r-1}{3 r-1} R_{12}+\frac{r}{3 r-1} Z_{k}=\lambda_{12} W_{1}+\lambda_{21} W_{2} .
$$

Let $d_{i_{1} i_{2} i_{3}}, i_{1}+i_{2}+i_{3}=2 r-1$, be the Bézier ordinates of $p$ related to this subtriangle $\hat{\mathcal{T}}_{k}$. By Lemma 4.1 we know that a subset of these ordinates in the neighbourhood of $W_{1}$ can be computed by subdividing a particular Bernstein basis polynomial of degree $2 r-1$ defined over PSr-triangle $t_{1}$. Let $\mathcal{B}_{2 r-1}$ be the blossom of this Bernstein basis polynomial, and let $\sigma^{l}=\left(\sigma_{1}^{l}, \sigma_{2}^{l}, \sigma_{3}^{l}\right), l=1,2,3$, be the barycentric coordinates with respect to $t_{1}$ of the points $V_{1}, U_{12}$ and $U_{13}$, respectively, where

$$
U_{12}=\frac{r}{3 r-1} V_{1}+\frac{2 r-1}{3 r-1} R_{12}, \quad U_{13}=\frac{r}{3 r-1} V_{1}+\frac{2 r-1}{3 r-1} Z_{k} .
$$

Then, the ordinates $d_{i_{1} i_{2} i_{3}}, i_{1}+i_{2}+i_{3}=2 r-1$ and $i_{1} \geq r$, can be written as

$$
d_{i_{1} i_{2} i_{3}}=\mathcal{B}_{2 r-1}(\underbrace{\sigma^{1}, \ldots, \sigma^{1}}_{i_{1}-r \text { times }}, \underbrace{\sigma^{2}, \ldots, \sigma^{2}}_{i_{2} \text { times }}, \underbrace{\sigma^{3}, \ldots, \sigma^{3}}_{i_{3}+r \text { times }}) \text {. }
$$

Let $\varrho^{l}=\left(\varrho_{1}^{l}, \varrho_{2}^{l}, \varrho_{3}^{l}\right), l=1,2,3$, be the barycentric coordinates of the points $W_{l}$ with respect to subtriangle $\hat{\mathcal{T}}_{k}\left(W_{1}, T_{12}, Z_{k}\right)$. Using (4.9), we have

$$
\varrho^{1}=(1,0,0), \quad \varrho^{2}=\left(\frac{-\lambda_{12}}{\lambda_{21}}, \frac{1}{\lambda_{21}}, 0\right), \quad \varrho^{3}=\left(\frac{z_{2} \lambda_{12}}{z_{3} \lambda_{21}}-\frac{z_{1}}{z_{3}},-\frac{z_{2}}{z_{3} \lambda_{21}}, \frac{1}{z_{3}}\right) .
$$

Let $\mathcal{P}_{2 r-1}$ be the blossom of polynomial $p$ with respect to $\hat{\mathcal{T}}_{k}$. Then, by $(2.10)$, we can write the Bézier ordinates $b_{i_{1} i_{2} i_{3}}, i_{1}+i_{2}+i_{3}=2 r-1$, of $p$ with respect to $\tilde{\mathcal{T}}_{k}$ in terms of the ordinates $d_{i_{1} i_{2} i_{3}}$ :

$$
b_{i_{1} i_{2} i_{3}}=\mathcal{P}_{2 r-1}(\underbrace{\varrho^{1}, \ldots, \varrho^{1}}_{i_{1} \text { times }}, \underbrace{\varrho^{2}, \ldots, \varrho^{2}}_{i_{2} \text { times }}, \underbrace{\varrho^{3}, \ldots, \varrho^{3}}_{i_{3} \text { times }}) .
$$

Because $\varrho^{1}=(1,0,0)$, only the ordinates $d_{i_{1} i_{2} i_{3}}$ with $i_{1} \geq r$ are involved in the expressions (4.12) of the ordinates $b_{i_{1} i_{2} i_{3}}$ with $i_{1} \geq r$. Using Theorem 2.2 together with (4.10) and (4.12), we obtain that for $i_{1} \geq r$

$$
b_{i_{1} i_{2} i_{3}}=\mathcal{B}_{2 r-1}(\underbrace{\sigma^{1}, \ldots, \sigma^{1}}_{i_{1}-r \text { times }}, \underbrace{\sigma^{3}, \ldots, \sigma^{3}}_{r \text { times }}, \underbrace{\sum_{l=1}^{2} \varrho_{l}^{2} \sigma^{l}, \ldots, \sum_{l=1}^{2} \varrho_{l}^{2} \sigma^{l}}_{i_{2} \text { times }}, \underbrace{\sum_{l=1}^{3} \varrho_{l}^{3} \sigma^{l}, \ldots, \sum_{l=1}^{3} \varrho_{l}^{3} \sigma^{l}}_{i_{3} \text { times }}) .
$$

By the definition of $\sigma^{l}$ and $\varrho^{l}$, we find after some elementary calculations that $\sum_{l=1}^{2} \varrho_{l}^{2} \sigma^{l}$ are the barycentric coordinates with respect to $\mathrm{PS} r$-triangle $t_{1}$ of the PSr-point

$$
\varrho_{1}^{2} V_{1}+\varrho_{2}^{2} U_{12}=\frac{-\lambda_{12}}{\lambda_{21}} V_{1}+\frac{1}{\lambda_{21}} U_{12}=\frac{r}{3 r-1} V_{1}+\frac{2 r-1}{3 r-1} V_{2}=S_{12},
$$

and that $\sum_{l=1}^{3} \varrho_{l}^{3} \sigma^{l}$ are the barycentric coordinates of the PSr-point

$$
\varrho_{1}^{3} V_{1}+\varrho_{2}^{3} U_{12}+\varrho_{3}^{3} U_{13}=\left(\frac{z_{2} \lambda_{12}}{z_{3} \lambda_{21}}-\frac{z_{1}}{z_{3}}\right) V_{1}-\frac{z_{2}}{z_{3} \lambda_{21}} U_{12}+\frac{1}{z_{3}} U_{13}=\frac{r}{3 r-1} V_{1}+\frac{2 r-1}{3 r-1} V_{3}=S_{13} .
$$


By the construction of PSr-triangle $t_{1}$, we know that $t_{1}$ contains the PSr-points $V_{1}, S_{12}$ and $S_{13}$. One can also easily see that the points $U_{12}$ and $U_{13}$ are inside $t_{1}$. Thus, the barycentric coordinates of these points with respect to $t_{1}$ are all nonnegative. Each Bernstein basis polynomial is nonnegative on its domain triangle, and its blossom $\mathcal{B}_{2 r-1}\left(\tau^{1}, \ldots, \tau^{2 r-1}\right)$ is nonnegative whenever the barycentric coordinates $\tau^{l}, l=1, \ldots, 2 r-1$, are nonnegative. From (4.13) it follows that the Bézier ordinates $b_{i_{1} i_{2} i_{3}}\left(i_{1} \geq r\right)$ of polynomial $p$ are nonnegative when PSr-triangle $t_{1}$ contains the points $V_{1}, S_{12}$ and $S_{13}$. The remaining $b_{i_{1} i_{2} i_{3}}\left(i_{1}<r\right)$ are known to be zero. Thus, polynomial $p$ is nonnegative on $\tilde{\mathcal{T}}_{k}$.

Using subdivision rules (2.10), we can conclude that the Bézier ordinates of the Powell-Sabin B-spline $B_{1, j_{1} j_{2} j_{3}}^{v, r}(x, y)$ in the disk $D_{2 r-1}\left(Z_{k}\right)$ are nonnegative as well.

Lemma 4.3. Let $\mathcal{T}_{k}\left(V_{1}, V_{2}, V_{3}\right)$ be a macro-triangle in the molecule of vertex $V_{1}$, and consider the Bernstein-Bézier representation of a Powell-Sabin B-spline $B_{1, j_{1} j_{2} j_{3}}^{v, r}(x, y)$ related to vertex $V_{1}$ on $\mathcal{T}_{k}$. For a given $0 \leq m \leq r$, let $E_{m}$ be the row of domain points at distance $m$ parallel to the edges $\left\langle V_{1}, R_{12}\right\rangle \cup\left\langle R_{12}, V_{2}\right\rangle$. Then, the Bézier ordinates of the B-spline corresponding to the central $4 r-1$ domain points on $E_{m}$ can be regarded as the Bézier ordinates (after subdivision) of a onedimensional nonnegative polynomial $\hat{p}$ of degree $2 r-1$ defined over the line segment between the points $\hat{W}_{1}$ and $\hat{W}_{2}$, given in (3.7). These Bézier ordinates are nonnegative.

Proof. We use a similar line of argument as in the proof of Lemma 4.2. From the properties of Bernstein-Bézier polynomials and from the $C^{2 r-1}$-smoothness of the B-spline $B_{1, j_{1} j_{2} j_{3}}^{v, r}(x, y)$ across the interior edge $\left\langle R_{12}, Z_{k}\right\rangle$, it follows that the Bézier ordinates of the B-spline corresponding to the central $4 r-1$ domain points on $E_{m}$ can be regarded as the Bézier ordinates (after subdivision) of a onedimensional polynomial $\hat{p}$ of degree $2 r-1$. Let $\hat{b}_{i_{1} i_{2}}, i_{1}+i_{2}=2 r-1$, be the Bézier ordinates of this polynomial in Bernstein-Bézier form given on the line segment $\left\langle\hat{W}_{1}, \hat{W}_{2}\right\rangle$. By the construction of the B-spline, only the ordinates $\hat{b}_{i_{1} i_{2}}$ with $i_{1} \geq r$ in the neighbourhood of $\hat{W}_{1}$ are nonzero. The values of these nonzero ordinates are uniquely determined by the set $A_{1, j_{1} j_{2} j_{3}}$. We also consider the Bernstein-Bézier form of this polynomial on the smaller line segment $\left\langle\hat{W}_{1}, \hat{T}_{12}\right\rangle$, where

$$
\hat{T}_{12}=\frac{3 r-1-m}{3 r-1} R_{12}+\frac{m}{3 r-1} Z_{k}=\lambda_{12} \hat{W}_{1}+\lambda_{21} \hat{W}_{2} .
$$

Let $\hat{d}_{i_{1} i_{2}}, i_{1}+i_{2}=2 r-1$, be the Bézier ordinates of $\hat{p}$ related to this smaller line segment. Similarly to (4.10), we can show that ordinates $\hat{d}_{i_{1} i_{2}}$ with $i_{1} \geq r$ can be expressed in terms of the blossom of a particular Bernstein basis polynomial of degree $2 r-1$ defined over PS $r$-triangle $t_{1}$, i.e.,

$$
\hat{d}_{i_{1} i_{2}}=\mathcal{B}_{2 r-1}(\underbrace{\sigma^{1}, \ldots, \sigma^{1}}_{i_{1}-r \text { times }}, \underbrace{\sigma^{2}, \ldots, \sigma^{2}}_{i_{2}+r-m \text { times }}, \underbrace{\sigma^{3}, \ldots, \sigma^{3}}_{m \text { times }}) .
$$

Let $\hat{\mathcal{P}}_{2 r-1}$ be the blossom of polynomial $\hat{p}$ with respect to line segment $\left\langle\hat{W}_{1}, \hat{T}_{12}\right\rangle$. Using (2.10) and (4.11) we can write the ordinates $\hat{b}_{i_{1} i_{2}}, i_{1}+i_{2}=2 r-1$, as

$$
\hat{b}_{i_{1} i_{2}}=\hat{\mathcal{P}}_{2 r-1}(\underbrace{\varrho^{1}, \ldots, \varrho^{1}}_{i_{1} \text { times }}, \underbrace{\varrho^{2}, \ldots, \varrho^{2}}_{i_{2} \text { times }}) .
$$

Only the ordinates $\hat{d}_{i_{1} i_{2}}$ with $i_{1} \geq r$ are involved in the expressions (4.15) of the ordinates $\hat{b}_{i_{1} i_{2}}$ with $i_{1} \geq r$. Similarly to (4.13), we can show that

$$
\hat{b}_{i_{1} i_{2}}=\mathcal{B}_{2 r-1}(\underbrace{\sigma^{1}, \ldots, \sigma^{1}}_{i_{1}-r \text { times }}, \underbrace{\sigma^{2}, \ldots, \sigma^{2}}_{r-m \text { times }}, \underbrace{\sigma^{3}, \ldots, \sigma^{3}}_{m \text { times }}, \underbrace{\sum_{l=1}^{2} \varrho_{l}^{2} \sigma^{l}, \ldots, \sum_{l=1}^{2} \varrho_{l}^{2} \sigma^{l}}_{i_{2} \text { times }}),
$$


for $i_{1} \geq r$. In the proof of Lemma 4.2, we have already shown that $\sum_{l=1}^{2} \varrho_{l}^{2} \sigma^{l}$ are the barycentric coordinates of PSr-point $S_{12}$ with respect to PSr-triangle $t_{1}$. Thus, all arguments of the blossom in (4.16) are nonnegative. It follows that all Bézier ordinates $\hat{b}_{i_{1} i_{2}}$ of polynomial $\hat{p}$ are nonnegative.

Lemma 4.4. The Bézier ordinates corresponding to domain points in the disk $D_{2 r-1}\left(Z_{k}\right)$ of a $B$ spline $B_{k, j_{1} j_{2} j_{3}}^{t, r}(x, y)$ related to triangle $\mathcal{T}_{k}$ in Bernstein-Bézier form can be regarded as the Bézier ordinates (after subdivision) of the Bernstein basis polynomial $B_{r-1-j_{1}, r-1-j_{2}, r-1-j_{3}}^{2 r-1}(x, y)$ of degree $2 r-1$ defined on the triangle with vertices $W_{i}, i=1,2,3$, as given in (3.5). These Bézier ordinates are nonnegative.

Proof. From definition (4.7) and Theorem 2.3 (with $d_{1}=3 r-1, d_{2}=2 r-1$ and $\mu=r-2$ ) it follows that the Bézier ordinates corresponding to domain points in the disk $D_{r-2}\left(Z_{k}\right)$ are equal in the Bernstein-Bézier representation of both the B-spline $B_{k, j_{1} j_{2} j_{3}}^{t, r}(x, y)$ and the considered Bernstein basis polynomial of degree $2 r-1$. We also know that

$$
D_{x}^{a} D_{y}^{b} B_{r-1-j_{1}, r-1-j_{2}, r-1-j_{3}}^{2 r-1}\left(V_{i}\right)=0,
$$

for all $0 \leq a+b \leq r-1$ and $i=1,2,3$. From the definition of the Powell-Sabin B-spline and (4.17), it follows that the Bézier ordinates corresponding to domain points in the three disks $D_{r-1}\left(W_{i}\right)$, $i=1,2,3$, are equal to zero in both Bernstein-Bézier representations.

Following a similar reasoning as used in the proof of Theorem 3.1, we can conclude that the Bézier ordinates in the disk $D_{2 r-1}\left(Z_{k}\right)$ of the Powell-Sabin B-spline $B_{k, j_{1} j_{2} j_{3}}^{t, r}(x, y)$ can be regarded as Bézier ordinates (after subdivision) of the considered Bernstein basis polynomial of degree $2 r-1$.

From the subdivision rules (2.10) and the nonnegativity of a Bernstein basis polynomial over its domain triangle, it follows that the considered Bézier ordinates are nonnegative.

We now show that the Powell-Sabin B-splines have a local support and form a convex partition of unity.

Property 4.1. The B-splines $B_{i, j_{1} j_{2} j_{3}}^{v, r}(x, y), i=1, \ldots, n_{v}, j_{1}+j_{2}+j_{3}=2 r-1$, and the B-splines $B_{k, j_{1} j_{2} j_{3}}^{t, r}(x, y), k=1, \ldots, n_{t}, j_{1}+j_{2}+j_{3}=r-2$, have a local support.

Proof. By their definition, it is easy to see that a B-spline $B_{i, j_{1} j_{2} j_{3}}^{v, r}$ is zero outside the molecule $M_{i}$ of vertex $V_{i}$, and that a B-spline $B_{k, j_{1} j_{2} j_{3}}^{t, r}$ vanishes outside triangle $\mathcal{T}_{k}$.

Property 4.2. The B-splines $B_{i, j_{1} j_{2} j_{3}}^{v, r}(x, y), i=1, \ldots, n_{v}, j_{1}+j_{2}+j_{3}=2 r-1$, and the B-splines $B_{k, j_{1} j_{2} j_{3}}^{t, r}(x, y), k=1, \ldots, n_{t}, j_{1}+j_{2}+j_{3}=r-2$, form a partition of unity on $\Omega$, i.e.,

$$
\sum_{i=1}^{n_{v}} \sum_{j_{1}+j_{2}+j_{3}=2 r-1} B_{i, j_{1} j_{2} j_{3}}^{v, r}(x, y)+\sum_{k=1}^{n_{t}} \sum_{j_{1}+j_{2}+j_{3}=r-2} B_{k, j_{1} j_{2} j_{3}}^{t, r}(x, y)=1,
$$

for all $(x, y) \in \Omega$.

Proof. We consider a particular macro-triangle $\mathcal{T}_{k}\left(V_{1}, V_{2}, V_{3}\right)$ in $\Delta$ and we show that the equality (4.18) holds on this triangle. The same argument can be used for the other triangles in $\Delta$. We focus on the Bernstein-Bézier representation of the Powell-Sabin spline in the left-hand side of (4.18) and we verify that all Bézier ordinates in this representation (see Figure 2) are equal to one. If all Bézier ordinates are equal to one, then the spline is also equal to one. 
From the definition of the Powell-Sabin B-splines it follows that only the $N^{v}$ basis functions related to vertex $V_{i}$ have function and derivative values at $V_{i}$ that are not all zero. We also know that the Bernstein basis polynomials used in definition (4.6) form a partition of unity on PSr-triangle $t_{i}$. We then immediately obtain that

$$
\sum_{j_{1}+j_{2}+j_{3}=2 r-1} \alpha_{i, j_{1} j_{2} j_{3}}^{00}=1
$$

and

$$
\sum_{j_{1}+j_{2}+j_{3}=2 r-1} \alpha_{i, j_{1} j_{2} j_{3}}^{a b}=0, \quad 1 \leq a+b \leq 2 r-1,
$$

for $i=1,2,3$. It follows that all Bézier ordinates in the three disks $D_{2 r-1}\left(V_{i}\right), i=1,2,3$, are equal to one.

From the construction of the Powell-Sabin spline we know that the Bézier ordinates in the disk $D_{2 r-1}\left(Z_{k}\right)$ around split point $Z_{k}$ can be regarded as Bézier ordinates after subdivision of a single polynomial $p$ of degree $2 r-1$ defined on the triangle $\tilde{\mathcal{T}}_{k}$ with vertices $W_{i}, i=1,2,3$, given in (3.5). Let $b_{i_{1} i_{2} i_{3}}, i_{1}+i_{2}+i_{3}=2 r-1$, be the Bézier ordinates in the Bernstein-Bézier representation of this polynomial $p$ on $\tilde{\mathcal{T}}_{k}$. By the construction of the Powell-Sabin spline, the values of $b_{i_{1} i_{2} i_{3}}$ in the three disks $D_{r-1}\left(W_{i}\right), i=1,2,3$, are specified by the values of $\alpha_{i, j_{1} j_{2} j_{3}}^{a b}$ in the sets $A_{i, j_{1} j_{2} j_{3}}$, $i=1,2,3$ and $j_{1}+j_{2}+j_{3}=2 r-1$. Because of (4.19), the ordinates $b_{i_{1} i_{2} i_{3}}$, for each $i_{1} \geq r$ or $i_{2} \geq r$ or $i_{3} \geq r$, must be equal to one. We also know that the Powell-Sabin B-splines with respect to the vertices do not influence the values of the other ordinates $b_{i_{1} i_{2} i_{3}}$ with $i_{1}<r$ and $i_{2}<r$ and $i_{3}<r$. Lemma 4.4 implies that the Powell-Sabin B-splines (in Bernstein-Bézier representation) with respect to triangle $\mathcal{T}_{k}$ can be locally regarded as a Bernstein basis polynomial of degree $2 r-1$ defined on the smaller triangle $\tilde{\mathcal{T}}_{k}$. By taking the sum over these Bernstein basis polynomials we obtain that also the ordinates $b_{i_{1} i_{2} i_{3}}$, with $i_{1}<r$ and $i_{2}<r$ and $i_{3}<r$, of polynomial $p$ must be equal to one. Since all Bézier ordinates of $p$ are equal to one, it follows that the Bernstein-Bézier representation of the Powell-Sabin spline in the left-hand side of (4.18) has Bézier ordinates in the disk $D_{2 r-1}\left(Z_{k}\right)$ equal to one.

For each edge $e_{i j}=\left\langle V_{i}, V_{j}\right\rangle$ of $\mathcal{T}_{k}$, the remaining Bézier ordinates on the rows $0, \ldots, r-1$ parallel to $e_{i j}$ are determined by means of the $C^{2 r-1}$-smoothness conditions across the edges $\left\langle R_{i j}, Z_{k}\right\rangle$. Because we already know that the Bézier ordinates in the three disks $D_{2 r-1}\left(V_{i}\right)$ are equal to one, these remaining Bézier ordinates must also be equal to one.

Property 4.3. The B-splines $B_{i, j_{1} j_{2} j_{3}}^{v, r}(x, y), i=1, \ldots, n_{v}, j_{1}+j_{2}+j_{3}=2 r-1$, and the B-splines $B_{k, j_{1} j_{2} j_{3}}^{t, r}(x, y), k=1, \ldots, n_{t}, j_{1}+j_{2}+j_{3}=r-2$, are nonnegative.

Proof. We first prove that a B-spline $B_{i, j_{1} j_{2} j_{3}}^{v, r}(x, y)$ with respect to vertex $V_{i}$ is nonnegative. Because of the local support we only have to show nonnegativity over the molecule $M_{i}$ of vertex $V_{i}$. For the sake of simplicity, let $i=1$ and let $\mathcal{T}_{k}\left(V_{1}, V_{2}, V_{3}\right)$ be a triangle in the molecule $M_{1}$. We consider the Bernstein-Bézier representation of the B-spline on macro-triangle $\mathcal{T}_{k}$, see Figure 3(a). By Lemma 4.1 we know that the Bézier ordinates in the disk $D_{2 r-1}\left(V_{1}\right)$ are nonnegative. Lemma 4.2 implies that the ordinates in the disk $D_{2 r-1}\left(Z_{k}\right)$ are nonnegative. From Lemma 4.3 it follows that the Bézier ordinates on the rows $0,1, \ldots, r-1$ parallel to edge $\left\langle V_{1}, V_{2}\right\rangle$ are nonnegative, and in an analogous way one can prove that the Bézier ordinates on the rows $0,1, \ldots, r-1$ parallel to edge $\left\langle V_{1}, V_{3}\right\rangle$ are also nonnegative. By the construction of the B-spline, the remaining Bézier ordinates are zero. Since all its Bézier ordinates are nonnegative, the B-spline $B_{i, j_{1} j_{2} j_{3}}^{v, r}(x, y)$ is also nonnegative on $\mathcal{T}_{k}$. A similar argument can be used for all other triangles in the molecule $M_{1}$.

We now prove the nonnegativity of a B-spline $B_{k, j_{1} j_{2} j_{3}}^{t, r}(x, y)$ with respect to triangle $\mathcal{T}_{k}\left(V_{1}, V_{2}, V_{3}\right)$. By the local support of the B-spline, it is sufficient to show nonnegativity over $\mathcal{T}_{k}$. We consider 
the Bernstein-Bézier representation of the B-spline on macro-triangle $\mathcal{T}_{k}$, see Figure 3(b). By the construction of the spline, the spline has only nonzero Bézier ordinates corresponding to domain points in the disk $D_{2 r-1}\left(Z_{k}\right)$. By Lemma 4.4 these Bézier ordinates can be regarded as the Bézier ordinates after subdivision of a particular Bernstein basis polynomial of degree $2 r-1$. Since these Bézier ordinates are nonnegative, the B-spline $B_{k, j_{1} j_{2} j_{3}}^{t, r}(x, y)$ is nonnegative on $\mathcal{T}_{k}$ as well.

\section{Applications}

In this section we first show how control polynomials and control points can be defined. Then, a stable computation of the Bernstein-Bézier representation of a PSr-spline is given.

\subsection{Control polynomials and control points}

Each Powell-Sabin B-spline $B_{i, j_{1} j_{2} j_{3}}^{v, r}(x, y), j_{1}+j_{2}+j_{3}=2 r-1$, with respect to vertex $V_{i}$ is related to a Bernstein basis polynomial of degree $2 r-1$, see (4.6). The corresponding spline coefficients $c_{i, j_{1} j_{2} j_{3}}^{v, r}, j_{1}+j_{2}+j_{3}=2 r-1$, of the Powell-Sabin spline in representation (4.1) can be regarded as the Bézier ordinates of a polynomial of degree $2 r-1$ defined on PSr-triangle $t_{i}$. This polynomial will be called the control polynomial with respect to vertex $V_{i}$, and will be denoted by $T_{i}(x, y)$ :

$$
T_{i}(x, y)=\sum_{j_{1}+j_{2}+j_{3}=2 r-1} c_{i, j_{1} j_{2} j_{3}}^{v, r} B_{j_{1} j_{2} j_{3}}^{2 r-1}(x, y),
$$

with $B_{j_{1} j_{2} j_{3}}^{2 r-1}(x, y)$ the Bernstein basis polynomials (2.3) of degree $2 r-1$ defined on PS $r$-triangle $t_{i}$. By the local support of the B-splines and by definitions (4.6) and (5.1), we have the following relation between a PSr-spline $s(x, y)$ and its control polynomial $T_{i}(x, y)$ at vertex $V_{i}$ :

$$
\begin{aligned}
D_{x}^{a} D_{y}^{b} s\left(V_{i}\right) & =\sum_{j_{1}+j_{2}+j_{3}=2 r-1} c_{i, j_{1} j_{2} j_{3}}^{v, r} \alpha_{i, j_{1} j_{2} j_{3}}^{a b} \\
& =\frac{\left(\begin{array}{c}
3 r-1 \\
a+b
\end{array}\right)}{\left(\begin{array}{c}
2 r-1 \\
a+b
\end{array}\right)}\left(\frac{2 r-1}{3 r-1}\right)^{a+b} \sum_{j_{1}+j_{2}+j_{3}=2 r-1} c_{i, j_{1} j_{2} j_{3}}^{v, r} D_{x}^{a} D_{y}^{b} B_{j_{1} j_{2} j_{3}}^{2 r-1}\left(V_{i}\right) \\
& =\frac{\left(\begin{array}{c}
3 r-1 \\
a+b
\end{array}\right)}{\left(\begin{array}{c}
2 r-1 \\
a+b
\end{array}\right)}\left(\frac{2 r-1}{3 r-1}\right)^{a+b} D_{x}^{a} D_{y}^{b} T_{i}\left(V_{i}\right) .
\end{aligned}
$$

Property 5.1. The control polynomial $T_{i}(x, y)$ is tangent to the Powell-Sabin spline $s(x, y)$ at vertex $V_{i}$.

Proof. Using (5.2), we have

$$
s\left(V_{i}\right)=T_{i}\left(V_{i}\right), \quad D_{x} s\left(V_{i}\right)=D_{x} T_{i}\left(V_{i}\right), \quad D_{y} s\left(V_{i}\right)=D_{y} T_{i}\left(V_{i}\right),
$$

implying that $T_{i}(x, y)$ is tangent to the spline at $V_{i}$.

Let $Q_{i, j_{1} j_{2} j_{3}}^{v, r}=\left(X_{i, j_{1} j_{2} j_{3}}^{v, r}, Y_{i, j_{1} j_{2} j_{3}}^{v, r}\right)$ and $Q_{k, j_{1} j_{2} j_{3}}^{t, r}=\left(X_{k, j_{1} j_{2} j_{3}}^{t, r}, Y_{k, j_{1} j_{2} j_{3}}^{t, r}\right)$ be points satisfying

$$
\sum_{i=1}^{n_{v}} \sum_{j_{1}+j_{2}+j_{3}=2 r-1} X_{i, j_{1} j_{2} j_{3}}^{v, r} B_{i, j_{1} j_{2} j_{3}}^{v, r}(x, y)+\sum_{k=1}^{n_{t}} \sum_{j_{1}+j_{2}+j_{3}=r-2} X_{k, j_{1} j_{2} j_{3}}^{t, r} B_{k, j_{1} j_{2} j_{3}}^{t, r}(x, y)=x,
$$


and

$$
\sum_{i=1}^{n_{v}} \sum_{j_{1}+j_{2}+j_{3}=2 r-1} Y_{i, j_{1} j_{2} j_{3}}^{v, r} B_{i, j_{1} j_{2} j_{3}}^{v, r}(x, y)+\sum_{k=1}^{n_{t}} \sum_{j_{1}+j_{2}+j_{3}=r-2} Y_{k, j_{1} j_{2} j_{3}}^{t, r} B_{k, j_{1} j_{2} j_{3}}^{t, r}(x, y)=y .
$$

We then define the PSr-control points as

$$
\begin{aligned}
\mathbf{c}_{i, j_{1} j_{2} j_{3}}^{v, r} & =\left(X_{i, j_{1} j_{2} j_{3}}^{v, r}, Y_{i, j_{1} j_{2} j_{3}}^{v, r}, c_{i, j_{1} j_{2} j_{3}}^{v, r}\right), \\
\mathbf{c}_{k, j_{1} j_{2} j_{3}}^{t, r} & =\left(X_{k, j_{1} j_{2} j_{3}}^{t, r}, Y_{k, j_{1} j_{2} j_{3}}^{t, r}, c_{k, j_{1} j_{2} j_{3}}^{t, r}\right),
\end{aligned}
$$

with $c_{i, j_{1} j_{2} j_{3}}^{v, r}$ and $c_{k, j_{1} j_{2} j_{3}}^{t, r}$ the B-spline coefficients in representation (4.1). Since the Powell-Sabin spline basis forms a convex partition of unity, it follows that the graph of spline (4.1) lies inside the convex hull of the control points (5.4).

Property 5.2. Let $Q_{i, 1}, Q_{i, 2}$ and $Q_{i, 3}$ be the three corner points of PSr-triangle $t_{i}$ corresponding to vertex $V_{i}$. Let $\mathcal{T}_{k}\left(V_{1}, V_{2}, V_{3}\right)$ be a macro-triangle in $\Delta$, containing the points $W_{l}, l=1,2,3$, as defined in (3.5). If the points $Q_{i, j_{1} j_{2} j_{3}}^{v, r}$ related to $V_{i}$ are taken as

$$
Q_{i, j_{1} j_{2} j_{3}}^{v, r}=\frac{j_{1}}{2 r-1} Q_{i, 1}+\frac{j_{2}}{2 r-1} Q_{i, 2}+\frac{j_{3}}{2 r-1} Q_{i, 3},
$$

for all $j_{1}+j_{2}+j_{3}=2 r-1$, and the points $Q_{k, j_{1} j_{2} j_{3}}^{t, r}$ related to $\mathcal{T}_{k}$ are taken as

$$
Q_{k, j_{1} j_{2} j_{3}}^{t, r}=\frac{r-1-j_{1}}{2 r-1} W_{1}+\frac{r-1-j_{2}}{2 r-1} W_{2}+\frac{r-1-j_{3}}{2 r-1} W_{3},
$$

for all $j_{1}+j_{2}+j_{3}=r-2$, then they satisfy (5.3).

Proof. We denote the spline in the left-hand side of (5.3a) by $s^{x}(x, y)$, and the spline in the lefthand side of (5.3b) by $s^{y}(x, y)$. We consider a particular macro-triangle $\mathcal{T}_{k}\left(V_{1}, V_{2}, V_{3}\right)$ in $\Delta$ and we show that the equalities in (5.3) hold on this triangle. A polynomial in Bernstein-Bézier form (2.2), where the Bézier ordinates are taken as the $x$-coordinates (or $y$-coordinates) of the corresponding Bézier domain points, is equal to the plane $z=x$ (or $z=y$ ). This is a necessary and sufficient condition. We now verify that the Bézier control points in the Bernstein-Bézier representation of $s^{x}(x, y)$ lie on the plane $z=x$, and that the Bézier control points in the Bernstein-Bézier representation of $s^{y}(x, y)$ lie on the plane $z=y$. It then follows that $s^{x}(x, y)=x$ and $s^{y}(x, y)=y$. By the choice (5.5) for $Q_{i, j_{1} j_{2} j_{3}}^{v, r}$ and by relation (4.6) at vertex $V_{i}=\left(x_{i}, y_{i}\right)$, we obtain

$$
\begin{aligned}
\sum_{j_{1}+j_{2}+j_{3}=2 r-1} X_{i, j_{1} j_{2} j_{3}}^{v, r} \alpha_{i, j_{1} j_{2} j_{3}}^{00}=x_{i}, & \sum_{j_{1}+j_{2}+j_{3}=2 r-1} Y_{i, j_{1} j_{2} j_{3}}^{v, r} \alpha_{i, j_{1} j_{2} j_{3}}^{00}=y_{i}, \\
\sum_{j_{1}+j_{2}+j_{3}=2 r-1}^{v, r} X_{i, j_{1} j_{2} j_{3}}^{v, r} \alpha_{i, j_{1} j_{2} j_{3}}^{10}=1, & \sum_{j_{1}+j_{2}+j_{3}=2 r-1} Y_{i, j_{1} j_{2} j_{3}}^{v, r} \alpha_{i, j_{1} j_{2} j_{3}}^{10}=0, \\
\sum_{j_{1}+j_{2}+j_{3}=2 r-1} X_{i, j_{1} j_{2} j_{3}}^{v, r} \alpha_{i, j_{1} j_{2} j_{3}}^{01}=0, & \sum_{j_{1}+j_{2}+j_{3}=2 r-1} Y_{i, j_{1} j_{2} j_{3}}^{v, r} \alpha_{i, j_{1} j_{2} j_{3}}^{01}=1,
\end{aligned}
$$

and

$$
\sum_{j_{1}+j_{2}+j_{3}=2 r-1} X_{i, j_{1} j_{2} j_{3}}^{v, r} \alpha_{i, j_{1} j_{2} j_{3}}^{a b}=0, \quad \sum_{j_{1}+j_{2}+j_{3}=2 r-1} Y_{i, j_{1} j_{2} j_{3}}^{v, r} \alpha_{i, j_{1} j_{2} j_{3}}^{a b}=0,
$$

for $2 \leq a+b \leq 2 r-1$. It follows that all Bézier control points of $s^{x}(x, y)$ in the three disks $D_{2 r-1}\left(V_{i}\right), i=1,2,3$, are on the plane $z=x$, and that all Bézier control points of $s^{y}(x, y)$ in the three disks $D_{2 r-1}\left(V_{i}\right)$ are on the plane $z=y$. 
By the $C^{2 r-1}$-smoothness of a Powell-Sabin spline of smoothness $r$ at split point $Z_{k}$, we know that its Bézier control points in the disk $D_{2 r-1}\left(Z_{k}\right)$ can be regarded as the Bézier control points after subdivision of a single polynomial $p$ of degree $2 r-1$ defined on the triangle $\tilde{\mathcal{T}}_{k}$ with vertices $W_{i}$, $i=1,2,3$, given in (3.5). These polynomials corresponding to $s^{x}(x, y)$ and $s^{y}(x, y)$ are denoted by $p^{x}$ and $p^{y}$, respectively. Let $\mathbf{b}_{i_{1} i_{2} i_{3}}^{x}, i_{1}+i_{2}+i_{3}=2 r-1$, be the Bézier control points of polynomial $p^{x}$ on triangle $\tilde{\mathcal{T}}_{k}$. From the construction of a PSr-spline and from (5.7), it follows that the control points $\mathbf{b}_{i_{1} i_{2} i_{3}}^{x}$, for each $i_{1} \geq r$ or $i_{2} \geq r$ or $i_{3} \geq r$, must lie on the plane $z=x$. We know that the Powell-Sabin B-splines with respect to the vertices cannot influence the values of the other control points $\mathbf{b}_{i_{1} i_{2} i_{3}}^{x}$ with $i_{1}<r$ and $i_{2}<r$ and $i_{3}<r$. Lemma 4.4 implies that the Powell-Sabin B-splines (in Bernstein-Bézier representation) with respect to triangle $\mathcal{T}_{k}$ can be locally regarded as a Bernstein basis polynomial of degree $2 r-1$ defined on the smaller triangle $\tilde{\mathcal{T}}_{k}$. From the choice (5.6) for $Q_{k, j_{1} j_{2} j_{3}}^{t, r}$ it then follows that also the control points $\mathbf{b}_{i_{1} i_{2} i_{3}}^{x}$, with $i_{1}<r$ and $i_{2}<r$ and $i_{3}<r$, lie on the plane $z=x$. Analogously, we can show that the Bézier control points $\mathbf{b}_{i_{1} i_{2} i_{3}}^{y}$ of polynomial $p^{y}$ lie on the plane $z=y$. Thus, the Bézier control points of $s^{x}(x, y)$ and $s^{y}(x, y)$ in the disk $D_{2 r-1}\left(Z_{k}\right)$ lie on the planes $z=x$ and $z=y$, respectively.

By the $C^{2 r-1}$-smoothness of the splines $s^{x}(x, y)$ and $s^{y}(x, y)$ across the interior edges $\left\langle R_{i j}, Z_{k}\right\rangle$, one can easily see that the remaining Bézier control points on the rows $0, \ldots, r-1$ parallel to the edges $\left\langle V_{i}, V_{j}\right\rangle$ of $\mathcal{T}_{k}$ must also lie on the planes $z=x$ and $z=y$, respectively.

We note that $\mathbf{c}_{i, j_{1} j_{2} j_{3}}^{v, r}, j_{1}+j_{2}+j_{3}=2 r-1$, related to vertex $V_{i}$ are the Bézier control points of the control polynomial $T_{i}(x, y)$. They can be organized in a triangular Bézier control net. The control points $\mathbf{c}_{k, j_{1} j_{2} j_{3}}^{t, r}, j_{1}+j_{2}+j_{3}=r-2$, related to triangle $\mathcal{T}_{k}$ can also be organized in a triangular net.

The control polynomials mimic the shape of the spline surface in the neighbourhood of their corresponding vertices. They can be used to locally model or change the shape of the spline surface in a predictable way. The control polynomials can be geometrically manipulated through the control points. Because of the local support of the B-splines, a change of a control polynomial (or control point) will only affect the spline patches related to a few triangles in the triangulation.

\subsection{Bézier ordinates of a PSr-spline}

The Bézier ordinates of a Powell-Sabin spline $s(x, y)$ in representation (4.1) can be computed in a stable way from its coefficients $c_{i, j_{1} j_{2} j_{3}}^{v, r}$ and $c_{k, j_{1} j_{2} j_{3}}^{t, r}$, using only convex combinations. We consider the Bernstein-Bézier representation of the spline restricted to a triangle $\mathcal{T}_{k}\left(V_{1}, V_{2}, V_{3}\right)$ as shown in Figure 1. The barycentric coordinates of the points indicated in this figure are given in (4.9).

Because of the $C^{2 r-1}$-smoothness of the PSr-spline at vertex $V_{1}$ and the local support of the Bsplines, the Bézier ordinates in the disk $D_{2 r-1}\left(V_{1}\right)$ are completely specified by the choice of control polynomial $T_{1}(x, y)$ corresponding to vertex $V_{1}$, see (5.1). In Lemma 4.1 it has been shown that the Bézier ordinates of the B-splines $B_{1, j_{1} j_{2} j_{3}}^{v, r}(x, y), j_{1}+j_{2}+j_{3}=2 r-1$, in the neighbourhood of vertex $V_{1}$ can be computed as the Bézier ordinates of the Bernstein basis polynomials of degree $2 r-1$ with respect to PS $r$-triangle $t_{1}$ after subdivision. Hence, the Bézier ordinates of the spline $s(x, y)$ in the disk $D_{2 r-1}\left(V_{1}\right)$ can be simply found by subdividing control polynomial $T_{1}(x, y)$, using (2.10), onto the two smaller triangles spanned by the four points

$$
V_{1}, \quad \lambda_{12} V_{1}+\lambda_{21} S_{12}, \quad \lambda_{13} V_{1}+\lambda_{31} S_{13}, \quad z_{1} V_{1}+z_{2} S_{12}+z_{3} S_{13}
$$

where $S_{12}$ and $S_{13}$ are PSr-points related to vertex $V_{1}$, see (4.5). Analogously, one finds that the Bézier ordinates of the spline $s(x, y)$ in the disks $D_{2 r-1}\left(V_{2}\right)$ and $D_{2 r-1}\left(V_{3}\right)$ can be computed through subdivision of the control polynomials $T_{2}(x, y)$ and $T_{3}(x, y)$ corresponding to vertices $V_{2}$ and $V_{3}$, respectively. 
By the $C^{2 r-1}$-smoothness of the spline $s(x, y)$ at split point $Z_{k}$, the Bézier ordinates in the disk $D_{2 r-1}\left(Z_{k}\right)$ are specified by a single polynomial $p$ of degree $2 r-1$ defined over the triangle $\tilde{\mathcal{T}}_{k}$ with the vertices $W_{i}, i=1,2,3$, given in (3.5). Let $b_{i_{1} i_{2} i_{3}}, i_{1}+i_{2}+i_{3}=2 r-1$, be the Bézier ordinates of this polynomial $p$ on $\tilde{\mathcal{T}}_{k}$. From the construction of the B-splines, it follows that

$$
b_{r-1-j_{1}, r-1-j_{2}, r-1-j_{3}}=c_{k, j_{1} j_{2} j_{3}}^{t, r},
$$

for all $j_{1}+j_{2}+j_{3}=r-2$. The ordinates $b_{i_{1} i_{2} i_{3}}$ with $i_{1} \geq r$ are completely specified by the choice of control polynomial $T_{1}(x, y)$. Following the same line of argument as in the proof of Lemma 4.2 to obtain (4.13), we find that these ordinates can be expressed in terms of the blossom of control polynomial $T_{1}(x, y)$ with as arguments a mixture of the barycentric coordinates of the PSr-points $S_{12}$ and $S_{13}$ and the points in (5.8). We can use the multi-affine de Casteljau algorithm, see (2.9), to evaluate this blossom. The ordinates $b_{i_{1} i_{2} i_{3}}$ with $i_{2} \geq r$ or $i_{3} \geq r$ can be computed in a similar way from the control polynomials $T_{2}(x, y)$ or $T_{3}(x, y)$, respectively. Then, the Bézier ordinates of the spline $s(x, y)$ in the disk $D_{2 r-1}\left(Z_{k}\right)$ can be computed through subdivision of this polynomial $p$.

Given $0 \leq m \leq r-1$, let $E_{m}$ be the row of domain points at distance $m$ parallel to the edges $\left\langle V_{1}, R_{12}\right\rangle \cup\left\langle R_{12}, V_{2}\right\rangle$. From the $C^{2 r-1}$-smoothness of the spline $s(x, y)$ across the interior edge $\left\langle R_{12}, Z_{k}\right\rangle$, it follows that the Bézier ordinates of the spline corresponding to the central $4 r-1$ domain points along row $E_{m}$ are specified by a single onedimensional polynomial $\hat{p}$ of degree $2 r-1$ defined over the line segment between the points $\hat{W}_{1}$ and $\hat{W}_{2}$, given in (3.7). Let $\hat{b}_{i_{1} i_{2}}$, $i_{1}+i_{2}=2 r-1$, be the Bézier ordinates of this polynomial $\hat{p}$ on $\left\langle\hat{W}_{1}, \hat{W}_{2}\right\rangle$. The ordinates $\hat{b}_{i_{1} i_{2}}$ with $i_{1} \geq r$ are completely specified by control polynomial $T_{1}(x, y)$. Following a similar reasoning as in the proof of Lemma 4.3 to obtain (4.16), we find that these ordinates can be expressed in terms of the blossom of control polynomial $T_{1}(x, y)$ with as arguments a mixture of the barycentric coordinates of PSr-point $S_{12}$ and the points given in (5.8). One can use the multi-affine de Casteljau algorithm to evaluate this blossom. The other ordinates $\hat{b}_{i_{1} i_{2}}$ with $i_{2} \geq r$ can be obtained in an analogous way from the control polynomial $T_{2}(x, y)$. Then, the unknown Bézier ordinates of the spline $s(x, y)$ on row $E_{m}$ can be computed through subdivision of this polynomial $\hat{p}$.

In a similar way one can also compute the remaining Bézier ordinates of the spline $s(x, y)$ on the rows $0,1, \ldots, r-1$ parallel to the edges $\left\langle V_{2}, V_{3}\right\rangle$ and $\left\langle V_{3}, V_{1}\right\rangle$ from the control polynomials $T_{1}(x, y)$, $T_{2}(x, y)$ and $T_{3}(x, y)$.

Only convex combinations are needed in the presented computation of all Bézier ordinates of the spline $s(x, y)$ given its B-spline coefficients. More details of this computation can be found in [11] in the case of a quintic Powell-Sabin spline with smoothness $r=2$.

Once the Bernstein-Bézier representation of the spline is known, evaluation or differentiation of the spline within each of the six subtriangles can then be performed using the de Casteljau algorithm (see, e.g., [2]).

\section{Reduced spline spaces}

In this section we propose a further reduction of the Powell-Sabin spline space (3.2)-(3.3). We will derive specific choices of the B-spline coefficients $c_{k, j_{1} j_{2} j_{3}}^{t, r}, k=1, \ldots, n_{t}$ and $j_{1}+j_{2}+j_{3}=r-2$, in representation (4.1), such that the resulting Powell-Sabin splines are still able to reproduce exactly polynomials up to degree $3 r-1$. In this way, their optimal approximation order will be retained with less degrees of freedom.

We consider a single macro-triangle $\mathcal{T}_{k}\left(V_{1}, V_{2}, V_{3}\right)$ in $\Delta$, on which we define a polynomial $q(x, y)$ of degree $3 r-1$ in Bernstein-Bézier form (2.2). Its Bézier ordinates are denoted by $d_{i_{1} i_{2} i_{3}}, i_{1}+$ $i_{2}+i_{3}=3 r-1$. We now derive conditions on the B-spline coefficients of a Powell-Sabin spline $s(x, y) \in S^{r}\left(\Delta^{*}\right)$ to be equal to this polynomial $q(x, y)$ on $\mathcal{T}_{k}$. To this end, we consider the 
restriction of control polynomial $T_{1}(x, y)$ onto the triangle with the $\mathrm{PS} r$-points $V_{1}, S_{12}$ and $S_{13}$ as its three vertices. We denote the Bézier ordinates of the control polynomial on this finer triangle by $e_{1, j_{1} j_{2} j_{3}}, j_{1}+j_{2}+j_{3}=2 r-1$. Using (2.9) and (2.10), these ordinates can be easily computed from the spline coefficients $c_{1, j_{1} j_{2} j_{3}}^{v, r}$ related to vertex $V_{1}$. In this computation we only use convex combinations. From the definitions of PSr-spline $s(x, y)$ and control polynomial $T_{1}(x, y)$, it then follows that $s(x, y)$ interpolates polynomial $q(x, y)$ and its derivatives up to order $2 r-1$ at vertex $V_{1}$, i.e.,

$$
D_{x}^{a} D_{y}^{b} s\left(V_{1}\right)=D_{x}^{a} D_{y}^{b} q\left(V_{1}\right)
$$

for all $0 \leq a+b \leq 2 r-1$, if

$$
e_{1, j_{1}, j_{2}, j_{3}}=d_{r+j_{1}, j_{2}, j_{3}},
$$

for all $j_{1}+j_{2}+j_{3}=2 r-1$. We obtain similar interpolation conditions at the vertices $V_{2}$ and $V_{3}$. Let $e_{2, j_{1} j_{2} j_{3}}, j_{1}+j_{2}+j_{3}=2 r-1$, be the Bézier ordinates of control polynomial $T_{2}(x, y)$ restricted on the triangle with vertices $\left(V_{2}, S_{23}, S_{21}\right)$, and let $e_{3, j_{1} j_{2} j_{3}}, j_{1}+j_{2}+j_{3}=2 r-1$, be the Bézier ordinates of control polynomial $T_{3}(x, y)$ restricted on the triangle with vertices $\left(V_{3}, S_{31}, S_{32}\right)$. The spline $s(x, y)$ interpolates $q(x, y)$ and its derivatives up to order $2 r-1$ at the vertices $V_{2}$ and $V_{3}$, if for all $j_{1}+j_{2}+j_{3}=2 r-1$,

$$
e_{2, j_{1}, j_{2}, j_{3}}=d_{j_{3}, r+j_{1}, j_{2}}
$$

and

$$
e_{3, j_{1}, j_{2}, j_{3}}=d_{j_{2}, j_{3}, r+j_{1}}
$$

respectively.

Referring to Figure 2, the Bézier ordinates of spline $s(x, y)$ in the disk $D_{2 r-1}\left(Z_{k}\right)$ can be computed as the Bézier ordinates after subdivision of a polynomial $p(x, y)$ of degree $2 r-1$ defined on the triangle with vertices $W_{i}, i=1,2,3$, given in (3.5). The spline $s(x, y)$ has the same function value and derivative values up to order $2 r-1$ at split point $Z_{k}$ as polynomial $q(x, y)$, i.e.,

$$
D_{x}^{a} D_{y}^{b} s\left(Z_{k}\right)=D_{x}^{a} D_{y}^{b} q\left(Z_{k}\right)
$$

for all $0 \leq a+b \leq 2 r-1$, if the Bézier ordinates $b_{i_{1} i_{2} i_{3}}$ of $p(x, y)$ satisfy

$$
b_{i_{1} i_{2} i_{3}}=b_{i_{1}, i_{2}, i_{3}}^{[r]},
$$

for $i_{1}+i_{2}+i_{3}=2 r-1$. Here, the values of $b_{i_{1}, i_{2}, i_{3}}^{[r]}$ are obtained from $d_{i_{1} i_{2} i_{3}}$ by applying $r$ steps of the de Casteljau algorithm, i.e.,

$$
b_{i_{1}, i_{2}, i_{3}}^{[0]}=d_{i_{1} i_{2} i_{3}},
$$

for $i_{1}+i_{2}+i_{3}=3 r-1$, and

$$
b_{i_{1}, i_{2}, i_{3}}^{[m]}=z_{1} b_{i_{1}+1, i_{2}, i_{3}}^{[m-1]}+z_{2} b_{i_{1}, i_{2}+1, i_{3}}^{[m-1]}+z_{3} b_{i_{1}, i_{2}, i_{3}+1}^{[m-1]},
$$

for $i_{1}+i_{2}+i_{3}=3 r-m-1$, and $m=1, \ldots, r$. This can be shown as follows. Let $\mathcal{P}$ and $\mathcal{Q}$ be the blossoms of polynomials $p$ and $q$, respectively. By (6.6), we have

$$
\mathcal{P}\left(\tau^{1}, \tau^{2}, \ldots, \tau^{2 r-1}\right)=\mathcal{Q}(\underbrace{z, z, \ldots, z}_{r \text { times }}, \tau^{1}, \tau^{2}, \ldots, \tau^{2 r-1}),
$$

with $z=\left(z_{1}, z_{2}, z_{3}\right)$ the barycentric coordinates of split point $Z_{k}$ with respect to $\mathcal{T}_{k}$. Equality (6.7) can be easily verified by applying the multi-affine de Casteljau algorithm to both blossoms. From the subdivision property of blossoms (see (2.10)) and (6.7), it follows that the Bézier ordinates in the disk $D_{2 r-1}\left(Z_{k}\right)$ of a spline $s(x, y)$ satisfying (6.6) can be regarded as Bézier ordinates after subdivision of the polynomial $q(x, y)$. This implies (6.5). 
From (5.9) we know that the spline coefficients $c_{k, j_{1} j_{2} j_{3}}^{t, r}, j_{1}+j_{2}+j_{3}=r-2$, are equal to a specific subset of Bézier ordinates of polynomial $p(x, y)$. Using $(6.6)$ we get the constraints

$$
c_{k, j_{1} j_{2} j_{3}}^{t, r}=b_{r-1-j_{1}, r-1-j_{2}, r-1-j_{3}}^{[r]},
$$

for all $j_{1}+j_{2}+j_{3}=r-2$. From (6.6) and (6.8) it follows that a coefficient $c_{k, j_{1} j_{2} j_{3}}^{t, r}$ is only influenced by the ordinates $d_{i_{1} i_{2} i_{3}}$ of polynomial $q(x, y)$, where

$$
\begin{array}{ll}
r-1-j_{1} \leq i_{1} \leq 2 r-1-j_{1}, & \text { and } \\
r-1-j_{2} \leq i_{2} \leq 2 r-1-j_{2}, & \text { and } \\
r-1-j_{3} \leq i_{3} \leq 2 r-1-j_{3} . &
\end{array}
$$

Because $j_{1}+j_{2}+j_{3}=r-2$, it follows that at most the ordinates $d_{i_{1} i_{2} i_{3}}$, with

$$
1 \leq i_{1} \leq 2 r-1, \quad \text { and } \quad 1 \leq i_{2} \leq 2 r-1, \quad \text { and } \quad 1 \leq i_{3} \leq 2 r-1,
$$

are involved in any expression (6.8). Inspired by interpolation conditions (6.2)-(6.4) at the vertices, we can set the values $d_{i_{1} i_{2} i_{3}}$ in $(6.6 \mathrm{~b})$ as follows.

- If $i_{1} \leq 2 r-1, i_{2} \leq r-1$ and $i_{3} \leq r-1$, then

$$
d_{i_{1} i_{2} i_{3}}=e_{1, i_{1}-r, i_{2}, i_{3}} .
$$

- If $i_{1} \leq r-1, i_{2} \leq 2 r-1$ and $i_{3} \leq r-1$, then

$$
d_{i_{1} i_{2} i_{3}}=e_{2, i_{2}-r, i_{3}, i_{1}} .
$$

- If $i_{1} \leq r-1, i_{2} \leq r-1$ and $i_{3} \leq 2 r-1$, then

$$
d_{i_{1} i_{2} i_{3}}=e_{3, i_{3}-r, i_{1}, i_{2}} .
$$

- If $i_{1} \geq r, i_{2} \geq r$ and $i_{3} \geq 1$, then

$$
d_{i_{1} i_{2} i_{3}}=\tau_{1} e_{1, i_{1}-r, i_{2}, i_{3}}+\tau_{2} e_{2, i_{2}-r, i_{3}, i_{1}},
$$

given some values $\tau_{1} \geq 0, \tau_{2} \geq 0$ and $\tau_{1}+\tau_{2}=1$.

- If $i_{1} \geq r, i_{2} \geq 1$ and $i_{3} \geq r$, then

$$
d_{i_{1} i_{2} i_{3}}=\tau_{1} e_{1, i_{1}-r, i_{2}, i_{3}}+\tau_{3} e_{3, i_{3}-r, i_{1}, i_{2}},
$$

given some values $\tau_{1} \geq 0, \tau_{3} \geq 0$ and $\tau_{1}+\tau_{3}=1$.

- If $i_{1} \geq 1, i_{2} \geq r$ and $i_{3} \geq r$, then

$$
d_{i_{1} i_{2} i_{3}}=\tau_{2} e_{2, i_{2}-r, i_{3}, i_{1}}+\tau_{3} e_{3, i_{3}-r, i_{1}, i_{2}},
$$

given some values $\tau_{2} \geq 0, \tau_{3} \geq 0$ and $\tau_{2}+\tau_{3}=1$.

If the spline coefficients $c_{k, j_{1} j_{2} j_{3}}^{t, r}$ are chosen as in (6.8) with (6.6) and (6.9), then the corresponding Powell-Sabin spline $s(x, y)$ will be able to reproduce polynomials of degree $3 r-1$.

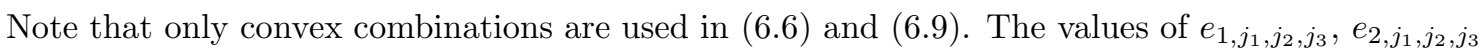
and $e_{3, j_{1}, j_{2}, j_{3}}$ can be found by subdivision of the control triangles. Thus, the proposed choice of the B-spline coefficients $c_{k, j_{1} j_{2} j_{3}}^{t, r}$ can be computed from the other B-spline coefficients $c_{i, j_{1} j_{2} j_{3}}^{v, r}$ through a sequence of convex combinations. It follows that the graph of such a reduced Powell-Sabin spline lies inside the convex hull of the control points $\mathbf{c}_{i, j_{1} j_{2} j_{3}}^{v, r}$.

A spline $s(x, y)$ in such a reduced spline space $S_{r e d}^{r}\left(\Delta^{*}\right)$ is uniquely defined by the following interpolation problem:

$$
D_{x}^{a} D_{y}^{b} s\left(V_{l}\right)=f_{x^{a} y^{b}, l}, \quad 0 \leq a+b \leq 2 r-1, \quad l=1, \ldots, n_{v},
$$

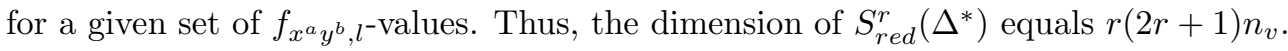




\section{Concluding remarks}

In this paper we have presented a family of bivariate (finite element) spline spaces with smoothness $r \geq 1$ and polynomial degree $d=3 r-1$. They are defined on a triangulation with Powell-Sabin refinement. We have also constructed a set of basis functions with some useful properties for computer aided geometric design. The spline spaces constructed in this paper are not of minimal polynomial degree for a given smoothness $r \geq 3$, see e.g. [3]. Yet, they have several other interesting properties.

1. PSr-splines can be uniquely defined by means of interpolation scheme (3.4) requiring function and derivative information at the vertices and split points. There is no need to know normal derivatives at certain points along edges. The reduced interpolation scheme (6.10) only requires function and derivative information at the vertices of the original triangulation.

2. PSr-splines can be represented in a normalized B-spline representation. The B-splines have a local support, they are nonnegative, and they form a partition of unity. The construction of the basis also allows the definition of control points and control polynomials, which are tangent to the spline surface. They may be useful for modelling purposes.

3. The Bernstein-Bézier form of such a spline can be computed from the PSr-spline coefficients using only convex combinations. In combination with the de Casteljau algorithm, this gives us a stable method to evaluate a PSr-spline.

Sablonnière constructed in [8] similar spline spaces with the same combination of smoothness $r$ and polynomial degree $d$. If the triangulation consists of a single triangle, then these spline spaces are identical to the ones presented in this paper. Yet, the construction slightly differs when the triangulation has more triangles. Sablonnière proposed an interpolation scheme requiring partial derivatives at the vertices and triangle split points, and also normal derivatives at points along the edges. He also suggested a family of spline spaces with reduced dimension.

The condition that each PSr-triangle must contain the PSr-points (4.5) is a sufficient condition for the nonnegativity of the basis functions. It is not a necessary condition: in case of quadratic Powell-Sabin splines $(r=1, d=2)$, Dierckx [1] has proposed another set of points, closer to the vertex.

For the reduced Powell-Sabin spline space presented in Section 6 one can also construct a normalized basis, such that its basis functions have a local support and form a convex partition of unity. Such a basis could be constructed in a similar way as the basis described in Section 4 for the full $\mathrm{PS} r$-spline space. A reduced basis function is defined by setting in the spline representation (4.1), with $c_{k, j_{1} j_{2} j_{3}}^{t, r}$ chosen as in Section 6 , a single coefficient $c_{i, j_{1} j_{2} j_{3}}^{v, r}$ to be one and all other coefficients $c_{i, j_{1} j_{2} j_{3}}^{v, r}$ to be zero.

\section{Acknowledgement}

Hendrik Speleers is a Postdoctoral Fellow of the Research Foundation Flanders (Belgium).

\section{References}

[1] P. Dierckx. On calculating normalized Powell-Sabin B-splines. Comput. Aided Geom. Design, 15:61-78, 1997. 
[2] G. Farin. Triangular Bernstein-Bézier patches. Comput. Aided Geom. Design, 3:83-127, 1986.

[3] M.J. Lai and L.L. Schumaker. Spline Functions on Triangulations. Encyclopedia of Mathematics and its Applications 110. Cambridge University Press, 2007.

[4] J. Maes and A. Bultheel. Stable multiresolution analysis on triangles for surface compression. Electr. Trans. Numer. Anal., 25:224-258, 2006.

[5] C. Manni and P. Sablonnière. Quadratic spline quasi-interpolants on Powell-Sabin partitions. Adv. Comput. Math., 26:283-304, 2007.

[6] M.J.D. Powell and M.A. Sabin. Piecewise quadratic approximations on triangles. ACM Trans. Math. Softw., 3:316-325, 1977.

[7] L. Ramshaw. Blossoming: a connect-the-dots approach to splines. Technical Report 19, Digital Systems Research Center, 1987.

[8] P. Sablonnière. Composite finite elements of class $C^{k}$. J. Comput. Appl. Math., 12\&13:541$550,1985$.

[9] P. Sablonnière. Error bounds for Hermite interpolation by quadratic splines on an $\alpha$ triangulation. IMA J. Numer. Anal., 7:495-508, 1987.

[10] H.P. Seidel. An introduction to polar forms. IEEE Comp. Graph. Appl., 13:38-46, 1993.

[11] H. Speleers. A normalized basis for quintic Powell-Sabin splines. Comput. Aided Geom. Design, 27:438-457, 2010.

[12] H. Speleers. A normalized basis for reduced Clough-Tocher splines. Comput. Aided Geom. Design, 27:700-712, 2010.

[13] H. Speleers, P. Dierckx, and S. Vandewalle. Numerical solution of partial differential equations with Powell-Sabin splines. J. Comput. Appl. Math., 189:643-659, 2006.

[14] H. Speleers, P. Dierckx, and S. Vandewalle. Quasi-hierarchical Powell-Sabin B-splines. Comput. Aided Geom. Design, 26:174-191, 2009. 